\title{
A lattice Boltzmann method for axisymmetric thermocapillary flows
}

\author{
Haihu $\operatorname{Liu}^{\mathrm{a}, *}$, Lei $\mathrm{Wu}^{\mathrm{b}}$, Yan $\mathrm{Ba}^{\mathrm{a}}$, Guang $\mathrm{Xi}^{\mathrm{a}}$ \\ ${ }^{a}$ School of Energy and Power Engineering, Xi'an Jiaotong University, 28 West Xianning Road, Xi'an 710049, China \\ ${ }^{b}$ James Weir Fluids Laboratory, Department of Mechanical $\& 3$ Aerospace Engineering, University of Strathclyde, Glasgow G1 \\ $1 X J, U K$
}

\begin{abstract}
In this work, we develop a two-phase lattice Boltzmann method (LBM) to simulate axisymmetric thermocapillary flows. This method simulates the immiscible axisymmetric two-phase flow by an improved color-gradient model, in which the single-phase collision, perturbation and recoloring operators are all presented with the axisymmetric effect taken into account in a simple and computational consistent manner. An additional lattice Boltzmann equation is introduced to describe the evolution of the axisymmetric temperature field, which is coupled to the hydrodynamic equations through an equation of state. This method is first validated by simulations of Rayleigh-Bénard convection in a vertical cylinder and thermocapillary migration of a deformable droplet at various Marangoni numbers. It is then used to simulate the thermocapillary migration of two spherical droplets in a constant applied temperature gradient along their line of centers, and the influence of the Marangoni number $(\mathrm{Ca})$, initial distance between droplets $\left(S_{0}\right)$, and the radius ratio of the leading to trailing droplets $(\Lambda)$ on the migration process is systematically studied. As $M a$ increases, the thermal wake behind the leading droplet strengthens, resulting in the transition of the droplet migration from coalescence to non-coalescence; and also, the final distance between droplets increases with $M a$ for the non-coalescence cases. The variation of $S_{0}$ does not change the final state of the droplets although it has a direct impact on the migration process. In contrast, $\Lambda$ can significantly influence the migration process of both droplets and their final state: at low $M a$, decreasing $\Lambda$ favors the coalescence of both droplets; at high $M a$, the two droplets do not coalesce eventually but migrate with the same velocity for the small values of $\Lambda$, and decreasing $\Lambda$ leads to a shorter equilibrium time and a faster migration velocity.
\end{abstract}

Keywords: Axisymmetric thermal flow, Thermocapillary migration, Lattice Boltzmann method, Marangoni number, Droplet interactions

\section{Introduction}

Thermocapillary convection is a phenomenon of fluid movement that arises as a consequence of the variation of interfacial tension at a fluid-fluid interface caused by temperature differences. It can be employed as a mechanism for driving the motion of droplets and bubbles immersed in a second fluid. For most fluids, the interfacial tension decreases with increasing temperature, and the induced thermocapillary stresses (also

\footnotetext{
* Corresponding author

Email address: haihu.liu@xjtu.edu.cn (Haihu Liu)
} 
called Marangoni stresses) lead to the migration of droplets or bubbles from the regions of low temperature, where the interfacial tension is high, to the warmer regions, where the interfacial tension is low. The thermocapillary migration of droplets and bubbles plays an important role in various industrial applications involving microgravity or microfluidic devices, where bulk phenomena can be negligible in comparison with interfacial effects due to large surface-to-volume ratio and low Reynolds number. To date, it has attracted an increasing amount of research interest worldwide along with the progress of human space exploration and microfluidic technologies.

The study on the thermocapillary migration of droplets or bubbles dates back to the pioneering work of Young et al. [1], who derived an analytical expression for the terminal migration velocity of an isolated spherical droplet in a constant temperature gradient by assuming that the convective transport of momentum and energy are negligible. Since then, extensive works on this subject have been conducted theoretically, experimentally and numerically, and most of them have been summarized in the review book by Subramanian and Balasubramaniam [2] as well as in the recent article by Yin and Li [3]. However, it is still challenging to conduct precise experimental measurements of the local temperature and flow fields during the migration process of droplets. Theoretical study based on the method of reflections has been used for predicting the motion of two well-separated droplets at an arbitrary orientation relative to the line of droplet centers [4]. Unfortunately, it is restricted to ideally spherical droplets and is unable to describe the deformation and coalescence of droplets. Numerical modelling and simulations can complement theoretical and experimental studies, providing an efficient pathway to enhance our understanding of the thermocapillary migration and interaction of droplets.

A variety of numerical methods have been proposed to simulate thermocapillary flows with deformed interfaces, and they can roughly be divided into two categories: one is the interface-tracking method, which uses the Lagrangian approach to explicitly represent the interface, such as the front-tracking method [5, 3], boundary-integral method [6], and immersed-boundary method [7]; and the other is the interface-capturing method, which uses an indicator function to implicitly represent the interface in an Eulerian grid, such as the volume-of-fluid (VOF) method [8], and level-set (LS) method [9]. However, the interface-tracking methods are not suitable for dealing with interface breakup and coalescence, because the interface must be manually ruptured based upon some $a d$-hoc criteria. The VOF and LS methods require interface reconstruction or reinitialization to represent or correct the interface, which may be complex or unphysical. Physically, the interface and its dynamical behavior are the natural consequence of microscopic interactions among fluid molecules. Thus, mesoscopic level methods may be better suited to simulate complex interfacial dynamics in a multiphase system.

The lattice Boltzmann method (LBM) is known to be capable of modeling interfacial interactions while incorporating fluid flow as a system feature. It is a pseudo-molecular method based on particle distribution functions that performs microscopic operations with mesoscopic kinetic equations and reproduces macroscopic behavior. The LBM has several advantages over traditional CFD methods such as the ability to be programmed on parallel computers and the ease in dealing with complex boundaries [10]. Besides, its kinetic nature provides many of the advantages of molecular dynamics, making the LBM particularly useful for 
simulating multiphase, multicomponent flows. A number of multiphase, multicomponent models have been proposed in the LBM community, and they can be classified into four major types: color-gradient model [11], phase-field-based model $[12,13,14]$, interparticle-potential model [15], and mean-field theory model [16]. These models have gained great success in simulating multiphase flow problems with a constant interfacial tension $[17,10]$. Based on the color-gradient model, we proposed the first LBM model to simulate thermocapillary flows, through which we for the first time demonstrated numerically that the droplet manipulation can be achieved through the thermocapillary forces induced by the laser heating [18]. This model was later extended to deal with fluid-surface interactions [19]. In addition, we developed two phase-field-based thermocapillary models with one focusing on high-density-ratio two-phase flows [20] and the other on modelling fluid-surface interactions [21]. The thermocapillary color-gradient model inherits a series of advantages of the model by Halliday and his coworkers [22, 23], such as low spurious velocities, high numerical accuracy, strict mass conservation for each fluid and good numerical stability for a broad range of fluid properties, and its three-dimensional (3D) version is capable of simulating the axisymmetric thermocapillary migration of two spherical droplets subject to a constant temperature gradient in an infinite domain, as considered in this work. Such a treatment, however, does not take the advantage of the axisymmetric property of the thermal flow and usually needs large computational costs. Alternatively, one can develop an axisymmetric version of the color-gradient LBM that allows for the solution of thermocapillary flows at the computational cost of a $2 \mathrm{D}$ simulation.

In this work, an axisymmetric two-phase LBM, developed on the basis of the Cartesian thermocapillary model of Liu et al. [18], is presented to simulate thermocapillary flows. This method simulates the axisymmetric two-phase flow through a multiple-relaxation-time (MRT) color-gradient model, in which the single-phase collision, perturbation and recoloring operators are all presented with the axisymmetric effect taken into account in a simple and computational consistent manner. An additional lattice Boltzmann equation is also introduced to describe the evolution of the axisymmetric temperature field, which is coupled to the interfacial tension by an equation of state. The capability and accuracy of this method are first tested by two benchmark cases, i.e. Rayleigh-Bénard convection in a vertical cylinder and thermocapillary migration of a deformable droplet at various Marangoni numbers. It is then used to simulate the thermocapillary migration of two spherical droplets subject to a constant temperature gradient along their line of centers, in which the influence of the Marangoni number, initial distance between the centers of two droplets, and the radius ratio of the leading to trailing droplets on the migration process is systematically investigated. To the best of our knowledge, the present method is the first axisymmetric thermocapillary LBM, and the study on the thermocapillary migration and interaction can provide useful suggestions and guidance for the design and optimization of the future space experiments.

\section{Numerical method}

In this section, we present an axisymmetric version of the color-gradient LBM for thermocapillary flows, and it is developed on the basis of our previous Cartesian model [18], in which the capillary and Marangoni forces are both modeled using the concept of the continuum surface force [24], and the temperature is solved 
by a passive scalar approach and coupled with the flowfield through an equation of state. In the color-gradient LBM, two sets of distribution functions $f_{i}^{R}$ and $f_{i}^{B}$ are introduced to represent the "red" and "blue" fluids. The total distribution function is defined by $f_{i}=f_{i}^{R}+f_{i}^{B}$, which undergoes a collision step as

$$
f_{i}^{\dagger}(\mathbf{x}, t)=f_{i}(\mathbf{x}, t)+\Omega_{i}(\mathbf{x}, t)+\Phi_{i}
$$

where $f_{i}(\mathbf{x}, t)$ is the total distribution function in the $i$-th velocity direction at the position $\mathbf{x}$ and time $t, f_{i}^{\dagger}$ is the post-collision distribution function, $\Omega_{i}$ is the single-phase collision operator, and $\Phi_{i}$ is the forcing term. The single-phase collision operator is designed to recover the correct macroscopic equations of incompressible axisymmetric flows in each single-phase region. For the axisymmetric flows with an axis in the $z$-direction, the single-phase collision operator is given by $[25,26]$

$$
\Omega_{i}(\mathbf{x}, t)=-\sum_{j}\left(\mathbf{M}^{-1} \mathbf{S M}\right)_{i j}\left[f_{j}(\mathbf{x}, t)-f_{j}^{e q}(\mathbf{x}, t)\right]+\delta_{t} h_{i}\left(\mathbf{x}+\mathbf{e}_{i} \delta_{t} / 2, t+\delta_{t} / 2\right),
$$

which adopts the MRT model [27] instead of the Bhatangar-Gross-Krook (BGK) approximation in order to enhance the numerical stability and reduce unphysical spurious velocities. In the above equation, $f_{i}^{e q}$ is the equilibrium distribution functions of $f_{i} ; \mathbf{M}$ is a transformation matrix; $\mathbf{S}$ is a diagonal relaxation matrix; and $h_{i}$ is a source term defined at the position $\left(\mathbf{x}+\mathbf{e}_{i} \delta_{t} / 2\right)$ and time $\left(t+\delta_{t} / 2\right)$, where $\delta_{t}$ is the time step, and $\mathbf{e}_{i}$ is the lattice velocity in the $i$-th direction. For the two-dimensional 9-velocity (D2Q9) model, $\mathbf{e}_{i}$ is defined as $\mathbf{e}_{0}=(0,0), \mathbf{e}_{1,3}=( \pm c, 0), \mathbf{e}_{2,4}=(0, \pm c), \mathbf{e}_{5,7}=( \pm c, \pm c)$, and $\mathbf{e}_{6,8}=(\mp c, \pm c)$, where $c=\delta_{x} / \delta_{t}$ with $\delta_{x}$ being the lattice spacing.

The equilibrium distribution function is obtained by a second order Taylor expansion of Maxwell-Boltzmann distribution with respect to the local fluid velocity $\mathbf{u}$ :

$$
f_{i}^{e q}=\rho w_{i}\left[1+\frac{\mathbf{e}_{i} \cdot \mathbf{u}}{c_{s}^{2}}+\frac{\left(\mathbf{e}_{i} \cdot \mathbf{u}\right)^{2}}{2 c_{s}^{4}}-\frac{\mathbf{u}^{2}}{2 c_{s}^{2}}\right],
$$

where $\rho=\rho_{R}+\rho_{B}$ is the total density with the subscripts ' $\mathrm{R}$ ' and 'B' referring to the red and blue fluids respectively, $c_{s}=\frac{1}{\sqrt{3}} c$ is the speed of sound, and $w_{i}$ is the weight factor given by $w_{0}=4 / 9, w_{1-4}=1 / 9$ and $w_{5-8}=1 / 36$.

The source term $h_{i}$ in Eq.(2) is introduced to account for the axisymmetric effect in the single-phase Navier-Stokes equations (NSEs), and it is given by $[26,28]$

$$
h_{i}=-w_{i} \frac{\rho u_{r}}{r}+\frac{1}{c_{s}^{2}} w_{i} e_{i \alpha} H_{\alpha},
$$

with

$$
H_{\alpha}=\frac{\mu\left(\partial_{r} u_{\alpha}+\partial_{\alpha} u_{r}\right)}{r}-\frac{2 \mu u_{r}}{r^{2}} \delta_{\alpha r}-\frac{\rho u_{\alpha} u_{r}}{r}
$$

where $\alpha=\{r, z\}, r$ is the coordinate in radial direction; $u_{\alpha}$ is the component of velocity in the $\alpha$ direction, $\delta_{\alpha \beta}$ is the Kronecker delta with two indices, and $\mu$ is the dynamic viscosity of the fluid mixture.

The spatial distribution of the two fluids is described using a color function (or phase-field function), which is defined as

$$
\rho^{N}(\mathbf{x}, t)=\frac{\rho_{R}(\mathbf{x}, t)-\rho_{B}(\mathbf{x}, t)}{\rho_{R}(\mathbf{x}, t)+\rho_{B}(\mathbf{x}, t)}, \quad-1 \leq \rho^{N} \leq 1 .
$$


In the LBM community, the concept of continuum surface force (CSF) was first used by Lishchuk et al. [22] to model the interfacial force with a constant interfacial tension, which was demonstrated to greatly reduce the spurious velocities and improve the isotropy of the interface. It was later extended by Liu and Zhang [18] to model the interfacial force with temperature-dependent interfacial tension and Marangoni stress. Following Liu and Zhang [18], the interfacial force in 3D Cartesian coordinate system reads as

$$
\mathbf{f}_{s}(\mathbf{x}, t)=-\frac{1}{2} \sigma(\nabla \cdot \mathbf{n}) \nabla \rho^{N}+\frac{1}{2}\left|\nabla \rho^{N}\right|(\mathbf{I}-\mathbf{n} \otimes \mathbf{n}) \cdot \nabla \sigma
$$

where $\sigma$ is an interfacial tension parameter, and $\mathbf{n}$ is the unit vector normal to the interface defined by $\mathbf{n}=\nabla \rho^{N} /\left|\nabla \rho^{N}\right|$. The first term on the right-hand side of Eq. (7) is the interfacial tension force and the second term is the Marangoni stress. In the axisymmetric case, there is an extra term in the interfacial force $\mathbf{f}_{s}$, that is

$$
\mathbf{f}_{s}(\mathbf{x}, t)=-\frac{1}{2} \sigma\left(\nabla_{c} \cdot \hat{\mathbf{n}}\right) \nabla_{c} \rho^{N}+\frac{1}{2}\left|\nabla_{c} \rho^{N}\right|\left[\nabla_{c} \sigma-\left(\hat{\mathbf{n}} \cdot \nabla_{c} \sigma\right) \hat{\mathbf{n}}\right]-\frac{1}{2} \sigma \frac{\hat{n}_{r}}{r} \nabla_{c} \rho^{N},
$$

where $\hat{\mathbf{n}}=\left(\hat{n}_{r}, \hat{n}_{z}\right)=\left(\frac{\partial_{r} \rho^{N}}{\sqrt{\left(\partial_{r} \rho^{N}\right)^{2}+\left(\partial_{z} \rho^{N}\right)^{2}}}, \frac{\partial_{z} \rho^{N}}{\sqrt{\left(\partial_{r} \rho^{N}\right)^{2}+\left(\partial_{z} \rho^{N}\right)^{2}}}\right)$, and $\nabla_{c}$ is the gradient in the cylindrical coordinates given by $\nabla_{c}=\left(\partial_{r}, \partial_{z}\right)$. It is noted in the above equation that the first three terms on the right-hand side are those adopted by the color-gradient model in two dimensions, and that the last term is the extra term responsible for the three dimensionality.

In a thermocapillary flow, an equation of state is required to relate the interfacial tension to the temperature, which may be linear or nonlinear. For the sake of simplicity, we only consider a linear relation between the interfacial tension and the temperature in this work, i.e.,

$$
\sigma(T)=\sigma_{r e f}+\sigma_{T}\left(T-T_{r e f}\right)
$$

where $T_{r e f}$ is the reference temperature, $\sigma_{r e f}$ is the interfacial tension at $T_{r e f}$, and $\sigma_{T}$ is the rate of change of interfacial tension with temperature, defined as $\sigma_{T}=\partial \sigma / \partial T$.

Substituting Eq. (9) into Eq. (8), we obtain the interfacial force as

$$
\mathbf{f}_{s}(\mathbf{x}, t)=-\frac{1}{2} \sigma\left(\nabla_{c} \cdot \hat{\mathbf{n}}\right) \nabla_{c} \rho^{N}+\frac{1}{2} \sigma_{T}\left|\nabla_{c} \rho^{N}\right|\left[\nabla_{c} T-\left(\hat{\mathbf{n}} \cdot \nabla_{c} T\right) \hat{\mathbf{n}}\right]-\frac{1}{2} \sigma \frac{\hat{n}_{r}}{r} \nabla_{c} \rho^{N}
$$

The interfacial force Eq. (10) is then incorporated into LBM through the body force model of Guo et al. [29], as previously done in the Cartesian version of color-gradient model [18, 19]. According to Guo et al. [29], the forcing term $\Phi_{i}$ that is applied to realize the interfacial tension and Marangoni effects, is written as

$$
\Phi_{i}(\mathbf{x}, t)=-\sum_{j}\left[\mathbf{M}^{-1}\left(\mathbf{I}-\frac{1}{2} \mathbf{S}\right) \mathbf{M}\right]_{i j} F_{j}(\mathbf{x}, t)
$$

with

$$
F_{i}=w_{i}\left[\frac{\mathbf{e}_{i}-\mathbf{u}}{c_{s}^{2}}+\frac{\left(\mathbf{e}_{i} \cdot \mathbf{u}\right) \mathbf{e}_{i}}{c_{s}^{4}}\right] \cdot \mathbf{f}_{s} \delta_{t},
$$

where $\mathbf{I}$ is a $9 \times 9$ unit matrix, and the local fluid velocity should be defined to incorporate the spatially varying interfacial force, i.e.,

$$
\rho(\mathbf{x}, t) \mathbf{u}(\mathbf{x}, t)=\sum_{k} \sum_{i} f_{i}^{k}(\mathbf{x}, t) \mathbf{e}_{i}+\frac{1}{2} \mathbf{f}_{s}(\mathbf{x}, t) \delta_{t} .
$$


The transformation matrix $\mathbf{M}$ is constructed by the Gram-Schmidt orthogonalization procedure from the discrete velocity set, and is given explicitly by [27]

$$
\mathbf{M} \equiv\left(\begin{array}{c}
\langle\rho| \\
\langle e| \\
\langle\varepsilon| \\
\left\langle j_{r}\right| \\
\left\langle q_{r}\right| \\
\left\langle j_{z}\right| \\
\left\langle q_{z}\right| \\
\left\langle p_{r r}\right| \\
\left\langle p_{r z}\right|
\end{array}\right)=\left(\begin{array}{ccccccccc}
1 & 1 & 1 & 1 & 1 & 1 & 1 & 1 & 1 \\
-4 & -1 & -1 & -1 & -1 & 2 & 2 & 2 & 2 \\
4 & -2 & -2 & -2 & -2 & 1 & 1 & 1 & 1 \\
0 & 1 & 0 & -1 & 0 & 1 & -1 & -1 & 1 \\
0 & -2 & 0 & 2 & 0 & 1 & -1 & -1 & 1 \\
0 & 0 & 1 & 0 & -1 & 1 & 1 & -1 & -1 \\
0 & 0 & -2 & 0 & 2 & 1 & 1 & -1 & -1 \\
0 & 1 & -1 & 1 & -1 & 0 & 0 & 0 & 0 \\
0 & 0 & 0 & 0 & 0 & 1 & -1 & 1 & -1
\end{array}\right),
$$

where the Dirac notation of bra $\langle\cdot|$ symbolizes the 9-dimensional row vector. With the transformation matrix $\mathbf{M}$, the particle distribution function $f_{i}$ can be projected onto the moment space through $m_{i}=\sum_{j} \mathbf{M}_{i j} f_{j}$, and the resulting nine moments are

$$
\mathbf{m} \equiv\left(m_{0}, m_{1}, \ldots, m_{8}\right)^{\mathrm{T}}=\left(\rho, e, \varepsilon, j_{r}, q_{r}, j_{z}, q_{z}, p_{r r}, p_{r z}\right)^{\mathrm{T}},
$$

where $e$ and $\varepsilon$ are related to the total energy and the energy square, $j_{r}$ and $j_{z}$ are the $r$-and $z$-components of the momentum, i.e. $j_{r}=\rho u_{r}$ and $j_{z}=\rho u_{z}, q_{r}$ and $q_{z}$ are the $r$ - and $z$-components of the energy flux, and $p_{r r}$ and $p_{r z}$ are related to the symmetric and traceless components of the stress tensor, respectively. The diagonal relaxation matrix $\mathbf{S}$ is defined as

$$
\mathbf{S}=\operatorname{diag}\left[s_{0}, s_{1}, s_{2}, s_{3}, s_{4}, s_{5}, s_{6}, s_{7}, s_{8}\right]
$$

where the elements $s_{i}$ are the relaxation rates associated with each $f_{i}$. The parameters $s_{0}, s_{3}$ and $s_{5}$ correspond to the conserved moments (i.e., $\rho, j_{r}$ and $j_{z}$ ) and have no effect on the derivation of the NSEs [30]. For simplicity, we choose $s_{0}=s_{3}=s_{5}=0 . s_{1}$ determines the bulk viscosity $\zeta$ through

$$
\zeta=\left(\frac{1}{s_{1}}-\frac{1}{2}\right) c_{s}^{2} \delta_{t}
$$

and it is considered as an adjustable parameter since the binary fluids are incompressible. $s_{7}$ and $s_{8}$ are related to the kinematic viscosity $\nu$ by

$$
s_{7}=s_{8}=\frac{1}{\tau}, \quad \text { and } \nu=\left(\tau-\frac{1}{2}\right) c_{s}^{2} \delta_{t} .
$$

Besides, symmetry requires that $s_{4}=s_{6}$. Consequently, three independent parameters $s_{1}, s_{2}$ and $s_{4}\left(=s_{6}\right)$ can be freely adjusted to enhance the stability of MRT model. Following the guidelines and suggestions in Ref. [27], we choose these free parameters as $s_{1}=1.63, s_{2}=1.14$, and $s_{4}=s_{6}=1.92$ in this study. It was also demonstrated that such a choice can effectively suppress spurious velocities in the vicinity of the contact line, resulting in an increased numerical accuracy in simulating contact angles [21].

Using the Chapman-Enskog multiscale expansion, Eq. (1) can be reduced to the axisymmetric NSEs in the low frequency, long wavelength limit with Eqs. (2)-(5), (11) and (12). The resulting equations are

$$
\partial_{t} \rho+\partial_{\beta}\left(\rho u_{\beta}\right)=-r^{-1} \rho u_{r},
$$




$$
\rho\left(\partial_{t} u_{\alpha}+u_{\beta} \partial_{\beta} u_{\alpha}\right)=-\partial_{\alpha} p+\partial_{\beta}\left[\mu\left(\partial_{\beta} u_{\alpha}+\partial_{\alpha} u_{\beta}\right)\right]+f_{s \alpha}+\frac{\mu\left(\partial_{r} u_{\alpha}+\partial_{\alpha} u_{r}\right)}{r}-\frac{2 \mu u_{r}}{r^{2}} \delta_{\alpha r}
$$

where $p=\rho c_{s}^{2}$ is the pressure, and $\mu=\rho \nu$ is the dynamic viscosity of the fluid mixture. Note that the present LBM suffers from the singularity at $r=0$, as can be seen from Eqs.(4), (5) and (10). This singularity does not affect the derivation of the axisymmetic NSEs, as the axisymmetric NSEs are singular at $r=0$ as well. In this study, the pure red and blue fluids are assumed to have equal densities. To allow for unequal viscosities of the two fluids, one can determine the viscosity of the fluid mixture by a harmonic mean [31]:

$$
\frac{1}{\mu\left(\rho^{N}\right)}=\frac{1+\rho^{N}}{2 \mu_{R}}+\frac{1-\rho^{N}}{2 \mu_{B}},
$$

where $\mu_{k}(k=R$ or $B)$ is the dynamic viscosity of fluid $k$. It has been shown that the choice of Eq. (21) can ensure a constant viscosity stress across the interface, resulting in a higher accuracy than other choices [31].

Although the forcing term (also known as perturbation step) generates the interfacial tension and Marangoni stress, it does not ensure the immiscibility of both fluids. To promote phase segregation and maintain a reasonable interface, the segregation (recoloring) algorithm of Latva-Kokko and Rothman [32] is used. It can overcome the lattice pinning problem and creates a symmetric distribution of particles around the interface so that unphysical spurious velocities can be further reduced. By extending the algorithm of Latva-Kokko and Rothman to the axisymmetric case, the post-segregation (recolored) distribution functions of the red and blue fluids are [28]

$$
\begin{aligned}
& f_{i}^{R \ddagger}(\mathbf{x}, t)=\frac{\rho_{R}}{\rho} f_{i}^{\dagger}(\mathbf{x}, t)+\beta \frac{\rho_{R} \rho_{B}}{\rho} w_{i} \frac{\mathbf{e}_{i} \cdot \nabla_{c} \rho^{N}}{\left|\mathbf{e}_{i}\right|\left|\nabla_{c} \rho^{N}\right|}, \\
& f_{i}^{B \ddagger}(\mathbf{x}, t)=\frac{\rho_{B}}{\rho} f_{i}^{\dagger}(\mathbf{x}, t)-\beta \frac{\rho_{R} \rho_{B}}{\rho} w_{i} \frac{\mathbf{e}_{i} \cdot \nabla_{c} \rho^{N}}{\left|\mathbf{e}_{i}\right|\left|\nabla_{c} \rho^{N}\right|},
\end{aligned}
$$

where $\beta$ is a free parameter associated with the interface thickness and should take a value between 0 and 1 in order to ensure non-negative distribution functions. In this study, $\beta$ is taken as 0.7 to maintain a steady interface and at the same time keep the interface as narrow as possible [23]. In addition, a previous study also showed that this choice is necessary to reproduce correct behavior of droplet dynamics [33].

After the recoloring step, the red and blue distribution functions propagate to the neighboring lattice nodes, known as propagation or streaming step:

$$
f_{i}^{k}\left(\mathbf{x}+\mathbf{e}_{i} \delta_{t}, t+\delta_{t}\right)=f_{i}^{k \ddagger}(\mathbf{x}, t), \quad k=R \text { or } B
$$

and the resulting distribution functions are then used to calculate the densities of both fluids, i.e. $\rho_{k}=\sum_{i} f_{i}^{k}$.

The temperature field is solved using the thermal axisymmetric LB model proposed by Li et al. [34]. The evolution equation for the temperature field is given by

$$
g_{i}\left(\mathbf{x}+\mathbf{e}_{i} \delta_{t}, t+\delta_{t}\right)-g_{i}(\mathbf{x}, t)=-\omega_{g}\left[g_{i}(\mathbf{x}, t)-g_{i}^{e q}(\mathbf{x}, t)\right]+\Psi_{i}(\mathbf{x}, t)
$$

where $g_{i}$ is the temperature distribution function, $g_{i}^{e q}$ is the equilibrium distribution function of $g_{i}$, and $\Psi_{i}$ is the source term. The relaxation parameter $\omega_{g}$ is defined by $\omega_{g}=\left[1+e_{i r} \tau_{g} \delta_{t} / r\right] /\left(\tau_{g}+0.5\right)$ [34], in which $\tau_{g}$ is the dimensionless relaxation time determined by the thermal diffusivity $k$. The equilibrium distribution function $g_{i}^{e q}$ is given by

$$
g_{i}^{e q}=w_{i} T\left(1+\frac{\mathbf{e}_{i} \cdot \mathbf{u}}{c_{s}^{2}}\right),
$$


where $T$ is the temperature calculated by

$$
T=\sum_{i} g_{i}
$$

Following Li et al. [34], the source term $\Psi_{i}$ is taken as

$$
\Psi_{i}=-\frac{u_{r}}{r} g_{i}^{e q} \delta_{t}
$$

With the macroscopic velocity given by Eq. (13), Eq. (24) can recover the macroscopic temperature equation in a cylindrical coordinate system, i.e.,

$$
\partial_{t} T+u_{\beta} \partial_{\beta} T=\partial_{\beta}\left(k \partial_{\beta} T\right)+\frac{k}{r} \partial_{r} T
$$

where the thermal diffusivity is given by $k=\tau_{g} c_{s}^{2} \delta_{t}$.

\subsection{Boundary conditions and evaluations of derivatives}

To study axisymmetric thermocapillary flows, the boundary conditions for the distribution functions $f_{i}^{R}$, $f_{i}^{B}$, and $g_{i}$ should be handled properly. As depicted in Fig. 1, $r=0$ represents the axis of symmetry, and the singularity will occur at $r=0$ because of the terms containing $r^{-1}$ in our LBM formulation. To avoid the singularity, we set the first lattice line at $r=0.5 \delta_{x}$ and apply the symmetry boundary condition to a ghost lattice line positioned at $r=-0.5 \delta_{x}$ :

$$
\begin{aligned}
& f_{1}^{R \ddagger}(P)=f_{3}^{R \ddagger}(Q), \quad f_{5}^{R \ddagger}(P)=f_{6}^{R \ddagger}(Q), \quad f_{8}^{R \ddagger}(P)=f_{7}^{R \ddagger}(Q), \\
& f_{1}^{B \ddagger}(P)=f_{3}^{B \ddagger}(Q), \quad f_{5}^{B \ddagger}(P)=f_{6}^{B \ddagger}(Q), \quad f_{8}^{B \ddagger}(P)=f_{7}^{B \ddagger}(Q), \\
& g_{1}^{\dagger}(P)=g_{3}^{\dagger}(Q), \quad g_{5}^{\dagger}(P)=g_{6}^{\dagger}(Q), \quad g_{8}^{\dagger}(P)=g_{7}^{\dagger}(Q),
\end{aligned}
$$

where $Q$ is an arbitrary node at the first fluid line; $P$ is the symmetric ghost node of $Q ; g_{i}^{\dagger}$ denotes the post-collision value of $g_{i}$, and it is given by

$$
g_{i}^{\dagger}(\mathbf{x}, t)=g_{i}(\mathbf{x}, t)-\omega_{g}\left[g_{i}(\mathbf{x}, t)-g_{i}^{e q}(\mathbf{x}, t)\right]+\Psi_{i}(\mathbf{x}, t) .
$$

At the solid wall, no-slip boundary condition and adiabatic boundary condition are both enforced using Ladd's halfway bounce-back scheme [35], which means the particles that hit the solid wall, then simply return back in the opposite direction where they came from. Specifically, as shown in Fig. 1, the unknown distributions at the fluid node $\mathbf{x}_{f}$ adjacent to the solid wall are determined by

$$
\begin{aligned}
& f_{i}^{R}\left(\mathbf{x}_{f}, t+\delta_{t}\right)=f_{i^{*}}^{R \ddagger}\left(\mathbf{x}_{f}, t\right), f_{i}^{B}\left(\mathbf{x}_{f}, t+\delta_{t}\right)=f_{i^{*}}^{B \ddagger}\left(\mathbf{x}_{f}, t\right), \\
& g_{i}\left(\mathbf{x}_{f}, t+\delta_{t}\right)=g_{i^{*}}^{\dagger}\left(\mathbf{x}_{f}, t\right) \quad \text { for } i=\{3,6,7\},
\end{aligned}
$$

where $\mathbf{e}_{i^{*}}=-\mathbf{e}_{i}$. The Dirichlet thermal boundary conditions are imposed by a general halfway bounceback scheme recently proposed by Zhang et al. [36]. Following their scheme, the unknown temperature distributions at the fluid node $\mathbf{x}_{f}$ adjacent to the solid wall which has a constant surface temperature $T_{w}$, are determined by

$$
g_{i}\left(\mathbf{x}_{f}, t+\delta_{t}\right)=-g_{i^{*}}^{\dagger}\left(\mathbf{x}_{f}, t\right)+2 w_{i^{*}} T_{w}
$$


The partial derivatives in the source term $h_{i}$ and the interfacial force $\mathbf{f}_{s}$ should be evaluated via suitable difference schemes. To minimize the discretization errors, the fourth-order isotropic finite-difference scheme,

$$
\partial_{\alpha} \phi(\mathbf{x})=\frac{1}{c_{s}^{2} \delta_{t}} \sum_{i} w_{i} \phi\left(\mathbf{x}+\mathbf{e}_{i} \delta_{t}\right) e_{i \alpha}
$$

is used to evaluate the derivatives of a variable $\phi$ at $\mathbf{x} \neq \mathbf{x}_{f}$; whereas at the fluid node $\mathbf{x}_{f}$ we impose the derivative terms to be zero in the evaluation of the interfacial force, and use the second-order difference schemes to evaluate the derivative terms in $h_{i}$, i.e.

$$
\partial_{r} \phi\left(\mathbf{x}_{f}\right)=-\frac{1}{3 \delta_{x}}\left[3 \phi\left(\mathbf{x}_{f}\right)+\phi\left(\mathbf{x}_{f}+\mathbf{e}_{3} \delta_{t}\right)\right], \quad \partial_{z} \phi\left(\mathbf{x}_{f}\right)=\frac{1}{2 \delta_{x}}\left[\phi\left(\mathbf{x}_{f}+\mathbf{e}_{2} \delta_{t}\right)-\phi\left(\mathbf{x}_{f}+\mathbf{e}_{4} \delta_{t}\right)\right],
$$

which is obtained on the basis of the zero velocity condition at the solid wall.

\section{Results and discussion}

\subsection{Model validation}

In this section, the proposed axisymmetic LBM is validated by two numerical tests, namely RayleighBénard convection in a vertical cylinder and thermocapillary migration of an isolated droplet. The first test is to validate the capability for simulating axisymmetric thermal flows in absence of interfacial effects, and the second one is to explicitly assess the thermocapillary coupling.

Rayleigh-Bénard convection in a vertical cylinder, where a single-phase fluid layer is heated from lower wall and cooled from upper wall with constant temperatures $T_{h}$ and $T_{l}$ respectively $\left(T_{h}>T_{l}\right)$, is a subject of longstanding interest due to its relevance to many atmospheric and industrial applications. This problem has been studied extensively by experiments and numerical methods [37, 38, 39, 40]. As shown in Fig. 2, the computational domain has an aspect ratio of $H / D=1 / 2$, where $H$ and $D$ are the height and diameter of the vertical cylinder. The setup of boundary conditions and initial temperature are the same as Ref. [38], i.e., $T_{h}=1, T_{l}=0$, and the lateral wall is adiabatic. No-slip conditions are applied at all walls.

The simulations are conducted in a $100 \times 100$ lattice domain, and the properties of the single-phase fluid are chosen as $\rho=1, \nu=0.07$, and $k=0.1$, yielding a Prandtl number of 0.7 . The Rayleigh number is set to be $R a=\frac{g \gamma\left(T_{h}-T_{l}\right) H^{3}}{\nu k}=5000$, where $\gamma$ is the thermal expansion coefficient and $g$ is the gravitational acceleration. With the Boussinesq assumption, the buoyancy force $\mathbf{G}$ is expressed as $\mathbf{G}=\left(0, \rho g \gamma\left(T-T_{m}\right)\right)$ with $T_{m}=\frac{T_{h}+T_{l}}{2}$, and it is introduced into the LBM in the same way as the interfacial force. Fig. 3 shows the velocity vectors and the contours of temperature field in the steady state. It is interestingly seen that two different flow patterns may appear, depending on the initial temperature field. Specifically, when an initial temperature is set to $T_{l}$ everywhere, an upflow in the center of the cylinder will occur (see Fig. 3(a)); on the other hand, when an initial temperature is set to $T_{h}$ everywhere, a downflow is induced in the center (see Fig. 3(b)). These two interesting phenomena were also observed both numerically and experimentally in the previous studies $[37,38,39,40]$ In addition, we quantify the maximum velocities in the flowfield and list them in Table. 1. By comparison, it is obvious that our simulation results are in good agreement with those obtained in Refs. [38, 40]. 
We then consider the thermocapillary migration of an isolated droplet in microgravity environment, which is caused by the nonuniform interfacial tension induced by the imposed temperature gradient. Thermocapillary migration was first analyzed by Young et al. [1] under the assumption of the creeping flows (vanishing Reynolds and Marangoni numbers), in which the convective transport of momentum and energy can be neglected in comparison with molecular transport of these quantities. They derived a theoretical expression for the terminal migration velocity (also known as YGB velocity) of a spherical droplet (red fluid) subject to a constant temperature gradient, $\nabla T_{\infty}$, in an unbounded fluid medium (blue fluid):

$$
U_{Y G B}=\frac{2 U}{\left(2+3 \mu_{R} / \mu_{B}\right)\left(2+k_{R} / k_{B}\right)} .
$$

where $U=\frac{-\sigma_{T}\left|\nabla T_{\infty}\right| R}{\mu_{B}}$ is the nominal thermocapillary velocity, and $R$ is the droplet radius. By choosing $U$ and $R$ as the characteristic velocity and length, the Reynolds number and the Marangoni number are defined by

$$
R e=\frac{\rho_{B} U R}{\mu_{B}}, \quad M a=\frac{U R}{k_{B}},
$$

which are commonly used to characterize the thermocapillary migration of droplets and bubbles. The Prandtl number is related to $M a$ and $\operatorname{Re}$ by $\operatorname{Pr}=M a / R e$.

A droplet of radius $R=20$ is placed inside a computational domain of $N_{r} \times N_{z}=5 R \times 20 R$. The center of droplet is initially located at $\left(r_{0}, z_{0}\right)=(0,10 R)$. We apply a symmetry boundary condition at $r=0$, and no-slip boundary conditions at all of the other boundaries. A linear temperature field is imposed in the $z$-direction, with $T=0$ on the lower wall and $T=40$ on the upper wall, resulting in $\nabla T_{\infty}=0.1$. In order to assess the accuracy of the proposed axisymmetric LBM, we first carry out the numerical simulation with the fluid properties of $\mu_{R}=\mu_{B}=0.2, k_{R}=k_{B}=0.2, T_{\text {ref }}=20, \sigma_{\text {ref }}=2 \times 10^{-3}$, and $\sigma_{T}=10^{-4}$. Using these values, the theoretical migration velocity of a spherical droplet $U_{Y G B}$ is $1.33 \overline{3} \times 10^{-4}$, and the Reynolds and Marangoni numbers are 0.1 . In the simulations, the migration velocity of droplet is calculated by

$$
u_{d}(t)=\frac{\int_{\rho^{N}>0} u_{z} r d r d z}{\int_{\rho^{N}>0} r d r d z}=\frac{\sum_{\mathbf{x}} u_{z}(\mathbf{x}, t) r(\mathbf{x}, t) N\left(\rho^{N}(\mathbf{x}, t)\right)}{\sum_{\mathbf{x}} r(\mathbf{x}, t) N\left(\rho^{N}(\mathbf{x}, t)\right)},
$$

where the function $N\left(\rho^{N}\right)$ is defined as

$$
N\left(\rho^{N}\right)= \begin{cases}1, & \text { if } \rho^{N}>0 \\ 0, & \text { otherwise. }\end{cases}
$$

Fig. 4 shows the time evolution of the computed migration velocity normalized by $U_{Y G B}$ in the test case of $R e=M a=0.1$. The dimensionless time is defined as $t^{*}=U t / R$. Evidently, our simulation result is in excellent quantitative agreement with the theoretical prediction (represented by the dashed line) since the effects of convective transport of momentum and energy are negligible in this test case.

In addition, we conduct numerical simulations to study the thermocapillary migration of a deformable droplet at large Marangoni numbers, for which analytical results are not available. Fig. 5 presents the time evolutions of the normalized migration velocity for four different values of $M a$, i.e., $M a=1,10,10^{2}$ and $10^{3}$, at a constant Reynolds number of 1 . Different values of $M a$ are achieved by adjusting $k_{B}$ whilst keeping $k_{R}=k_{B}$. We also choose $\mu_{R, B}=0.1, \sigma_{T}=-2.5 \times 10^{-4}$ and $\sigma_{r e f}=5 \times 10^{-3}$, and keep all the other physical 
properties the same as those in the above test case. For different values of $M a$, the migration velocity of the droplet increases roughly at the same speed in the early stage, which is caused by the initial conditions used in our numerical simulations, i.e., $\left.\mathbf{u}\right|_{t=0}=0$ and $\left.T\right|_{t=0}=z\left|\nabla T_{\infty}\right|$. After an initial increase, the migration velocity will directly reach a steady value when $M a$ is small, i.e., $M a=1$. However, there are obvious increase-decrease processes (i.e., oscillations) in the time-velocity plots for large values of $M a(M a \geq 10)$, and the number and amplitude of oscillations both increase with $M a$. In spite of the oscillations, the droplet migration can reach a steady state for all of the Marangoni numbers considered. It is evidenced that the terminal migration velocity decreases monotonically with $M a$, consistent with the previous theoretical and numerical findings in the case of non-deformable droplets or bubbles $[41,42,5]$. The dependence of the terminal migration velocity on $M a$ can be explained by the isotherms surrounding the droplet, which are shown in Fig. 6, where the temperature value is labeled on each contour. Obviously, the enhanced convective transport of energy with increasing $M a$ results in the wrapping of the isotherms around the front of the droplet (also, the thermal boundary layer in front of the droplet becomes increasingly thin), leading to a substantial reduction of the temperature gradient at the droplet interface. Small average temperature gradient at the interface will reduce the driving force for the droplet migration. Fig. 6 also depicts the corresponding velocity vectors in a coordinate system moving with the droplet centroid. Relative to the migrating droplet, the flow pattern within the droplet exhibits recirculation flow that is similar to the Hills spherical vortex [43]. It is clear that the vortex intensity weakens as $M a$ increases.

\subsection{Axisymmetric thermocapillary migration of two deformable droplets}

Having established the accuracy of the proposed axisymmetric color-gradient LBM, we use it to simulate the thermocapillary migration of two viscous droplets in a constant temperature gradient along their line of centers. As illustrated in Fig. 7, two red droplets with the radii of $R_{1}$ (trailing droplet) and $R_{2}$ (leading droplet) are surrounded by the blue fluid in a computational domain of $[0,160] \times[0,800]$. The trailing droplet is initially centered at $(0,120)$ and its radius $R_{1}=40 . S$ is the distance between the droplet centres, and $S_{0}$ is the initial distance. A constant temperature gradient is imposed in the $z$-direction by specifying $T=0$ at the lower wall and $T=80$ at the upper wall. All of the boundary conditions are the same as in the second test case shown above. The Reynolds number and the Marangoni number are defined as in Eq. (36), where the characteristic length is selected as the radius of the trailing droplet, and the characteristic velocity

$U=-\frac{\sigma_{T}\left|\nabla T_{\infty}\right| R_{1}}{\mu_{B}}$. In the following simulations, we take $R e=1.2, \sigma_{T}=-7.5 \times 10^{-5}$, and $\sigma_{r e f}=6 \times 10^{-3}$ at the reference temperature $T_{r e f}=12$; and also, both fluids are assumed to have equal viscosity and thermal diffusivity for simplicity. It is easy to know from Eq. (35) that that a larger droplet leads to a larger migration velocity, so there will be weaker interaction for $R_{2}>R_{1}$ because $S$ becomes bigger after the simulation is started. Hence, we only consider the case of $R_{2} \leq R_{1}$ throughout this study.

\subsubsection{The influence of $M a$}

We first study the influence of $M a$ on the thermocapillary migration and interaction of two unequalsized droplets. The sizes of both droplets and their initial distance are kept constant with $R_{2}=0.5 R_{1}$ and $S_{0}=2.5 R_{1}$. Four different values of $M a$ are considered, i.e. $M a=10,30,100$ and 200, which are 
achieved by varying solely $k_{R}$ and $k_{B}$. Fig. 8 shows the time evolution of the droplet migration velocities for $M a=100$, in which the cases with an isolated droplet are also plotted for comparison. When two droplets interact and migrate upwards, there are some differences in their migration velocities. Specifically, the trailing droplet initially undergoes a rapid acceleration and deceleration, forming a noticeable overshoot in velocity. By contrast, the leading droplet has a much smaller overshoot, and eventually evolves to the same velocity as the trailing droplet. In addition, the motion of the trailing droplet does not deviate from that of the big isolated droplet until $t^{*}=10$, but the motion of the leading droplet deviates from that of the small isolated droplet much earlier. As indicated in Fig. 8, the terminal velocity of the binary droplets is lower than that of the big isolated droplet, but higher than that of the small isolated droplet. Quantitatively speaking, the terminal velocity of the binary droplets is around 0.0709 , which is quite close to the previous result obtained by the front-tracking method (0.07, extracted from Fig.15 in Ref. [3]). This suggests that the present color-gradient LBM is able to simulate accurately axisymmetric thermocapillary flows even with droplet interactions.

Fig. 9 show the comparison of temperature fields between the binary droplets and isolated droplets when the droplet migration has reached the steady state at $M a=100$. For an isolated droplet (Fig. 9(a) and (c)), the isotherms accumulate around the droplet front, where the temperature gradient along the migration direction is relatively large. On the other hand, there is a long thermal wake behind the droplet, where the temperature gradient along the migration direction is relatively small. When two droplets migrate together, the isotherms between two droplets are denser than those behind the isolated small droplet, but sparser than in front of the isolated big droplet (see Fig. 9(b)). This could be explained as follows: (1) the thermal wake caused by the leading droplet lowers the temperature gradient inside the trailing droplet; (2) the accumulated isotherms around the front of the trailing droplet enhances the temperature gradient at the rear of the leading droplet. Since a higher temperature gradient leads to a larger driving force for the thermocapillary migration, it is expected that the terminal migration velocity of binary droplets lies between that of the small isolated droplet and that of the big isolated droplet, as previously shown in Fig. 8.

Two droplets do not coalesce and eventually migrate at the same speed for $M a=100$, but it is not true for all of the Marangoni numbers. Fig. 10 shows the time evolution of the droplet distance $S$ for the Marangoni numbers ranging from 10 to 200. For high Marangoni numbers, i.e. $M a \geq 100, S$ first decreases, and then increases until reaching a constant value $S_{F}$. This is because, compared to the leading droplet, the trailing droplet initially has a much larger acceleration but becomes slower after the overshoot (see, e.g., Fig. 8). Note that $S_{F}$ increases with $M a$, and it may be greater than $S_{0}$. For moderate Marangoni numbers, i.e. $M a=30, S$ keeps decreasing and finally reaches a constant value that is greater than $\left(R_{1}+R_{2}\right)$, implying that both droplets would not touch or merge together. For low Marangoni number, i.e. $M a=10, S$ keeps decreasing rapidly until both droplets coalesce. It is worth emphasizing in Fig. 10 that the evolution of $S$ is recorded only when the gap between droplets $G>0.1 R_{1}$, where $G \approx S-\left(R_{1}+R_{2}\right)$. Fig. 11 shows the snapshots of the droplet shapes and the isotherms around the droplets at $M a=10$. Before the coalescence occurs, the temperature distribution inside the droplets does not change much and is close to that of the surrounding fluid at the same height, which means that the driving force determined by the temperature 
gradient for each droplet is roughly the same as in isolated case (i.e., the interaction mechanism between two droplets is very weak). It is known that the big droplet migrates faster than the smaller one in isolated case. Therefore, the trailing droplet is able to catch up with the leading one, resulting in a coalescence of the two droplets. During the coalescence, the interface curvatures change remarkably near the coalescence region (liquid bridge), where large velocities are locally induced and lead to the reverse of the isotherms inside the leading droplet. Specifically, the warping direction of the isotherms inside the leading droplet changes from convex-up to convex-down. As the coalescence ends, a bigger droplet of nearly spherical shape is formed; the isotherms warp around the droplet front but the isotherm-gathering effect is not strong due to low $M a$.

\subsubsection{The influence of $S_{0}$}

The influence of $S_{0}$ is studied for a constant Marangoni number. First, $M a$ is fixed at 100 , and $S_{0}$ is varied from $2 R_{1}$ to $3 R_{1}$. All of the other parameters are kept the same as those in Section 3.2.1. Fig. 12 shows the time evolution of the droplet migration velocities at different $S_{0}$ for $M a=100$. For the trailing droplet, the early transient of the droplet migration is identical for various $S_{0}$ because of the same initial conditions (including the velocity and temperature fields and the position of the trailing droplet) used in these simulations. After the overshoot, the migration of the trailing droplet exhibits different characteristics with the variation of $S_{0}$ : a larger $S_{0}$ leads to a faster migration velocity. This is easy to be understandable because, when $S_{0}$ is large, the influence of the thermal wake from the leading droplet is weaker, the migration process of the trailing droplet is closer to that of the corresponding isolated one, and the trailing droplet migrates faster. On the other hand, for the leading droplet, a larger $S_{0}$ leads to a slower migration velocity in the early stage of the simulation, which is attributed to the weaker isotherm-gathering effects from the trailing droplet. When the equilibrium is reached, both the leading and trailing droplets migrate with almost the same velocity, and the values of the common velocity are identical for different $S_{0}$.

The evolution of the droplet migration velocities in Fig. 12 implies that the variation of $S_{0}$ does not affect the final distance between droplets, although it has a direct impact on the migration process. This can be clearly seen in Fig. 13, which plots the time evolution of $S$ at different $S_{0}$ for $M a=100$. It is also noticed in Fig. 13 that the droplet distance $S$ decreases faster with increasing $S_{0}$ in the early stage of the simulation, which is because a larger $S_{0}$ results in a faster migration velocity of the trailing droplet but a slower migration velocity of the leading droplet (see Fig. 12). When $S$ is large, the trailing droplet always migrates faster than the leading one, so even if $S_{0}$ is extremely large, the two droplets are able to reach their common velocity with a fixed distance equal to those obtained in Fig. 13. In addition, for a constant Marangoni number, which is not limited to $M a=100$, we find that the variation of $S_{0}$ does not affect the final states of both droplets. As an example, Fig. 14 displays the time evolution of $S$ with different $S_{0}$ at a typical Marangoni number of 10. Note that all the parameter are kept the same as those used in Fig. 13 except Ma. For each $S_{0}$, the distance between droplets decreases monotonously until the two droplets coalesce (see Fig. 14), and finally the coalesced droplet forms a nearly spherical shape and migrates with a constant velocity. Also, the terminal migration velocities of the coalesced droplet are almost identical for different values of $S_{0}$ (not shown). 


\subsubsection{The influence of the droplet radius ratio}

The droplet radius ratio is defined as $\Lambda=R_{2} / R_{1}$, and its influence is first studied for $M a=100$ and $S_{0}=2.5 R_{1}$. Four different values of $\Lambda$ are considered, i.e. $\Lambda=3 / 8,1 / 2,3 / 4$ and 1 , which are achieved by adjusting $R_{2}$ while keeping $R_{1}$ fixed.

Fig. 15 illustrates the time evolution of the droplet distance $S$ for various $\Lambda$ at $M a=100$ and $S_{0}=2.5 R_{1}$. For small $\Lambda$, i.e. $\Lambda=3 / 8$, the bigger trailing droplet dominates the interactions between two droplets, and the droplet motion reaches the equilibria (at which both droplets migrate at the same speed and a fixed $S$ ) quickly. When $\Lambda$ is increased to $1 / 2$, the droplets take a longer time to reach their common velocity. This is attributed to the fact that the bigger leading droplet has a larger impact on the droplet interactions, leading to a longer time required for the interplay between droplets. For $\Lambda \geq 3 / 4$, the two droplets cannot reach a common velocity before the front of the leading droplet reaches its limitation position $(z=800)$, above which the interfacial tension $\sigma$ is unphysically negative. Note that, for a finite $S$ at $\Lambda=1$, the leading droplet always moves faster than the trailing one, so $S$ keeps continuously increasing in the entire simulation (also see Fig. 17 below). In addition, we also record the time evolution of the droplet migration velocity for two smallest values of $\Lambda$ (i.e. $\Lambda=3 / 8$ and $1 / 2$ ), which is shown in Fig. 16. It is found that the final migration velocity is lower for larger $\Lambda$ (or a larger leading droplet), contrary to the result of isolated droplets that a larger droplet leads to a higher migration velocity in the final state. This can be explained as a result of the enhanced temperature gradient along the droplet interfaces due to a decrease of $S_{F}$ at smaller $\Lambda$ (Fig. 15).

We then study the influence of $\Lambda$ on the thermocapillary migration of two droplets at a lower Marangoni number of 10. All of the other parameters are kept the same as those in the above case of $M a=100$. Fig. 17 shows the time evolution of $S$ at $M a=10$ and $S_{0}=2.5 R_{1}$ for $\Lambda=3 / 8,1 / 2,3 / 4$ and 1 . One can observe three distinct states of the droplet motion, depending on the value of $\Lambda$. For $\Lambda \leq 1 / 2, S$ keeps increasing until the onset of the droplet coalescence; and finally the two droplets coalesce into a single droplet that migrates with a constant velocity. For $\Lambda=3 / 4$, the two droplets interact, with a decreasing gap between them, and eventually reach the same velocity (i.e., $S$ becomes a constant). For $\Lambda=1, S$ keeps increasing because the leading droplet always migrates faster than the trailing one due to the isotherm-gathering effect above the trailing droplet. Based on the above discussions, it can be concluded that increasing $\Lambda$ decreases the possibility of droplet coalescence.

\section{Conclusions}

Based on the Cartesian model of Liu and Zhang [18], a lattice Boltzmann method is developed for the simulation of axisymmetric thermocapillary flows. Like the Cartesian version of thermocapillary model, this method solves the immiscible two-phase flows through a color-gradient model, which uses a collision operator consisting of three separate parts, namely the single-phase collision operator, perturbation operator, and the recoloring operator. The single-phase collision operator and the recoloring operator are essentially the same as those proposed in Ref. [28], in which the axisymmetric effects have been taken into account in a simple and computational consistent manner. In the perturbation step, the interfacial force of axisymmetric 
form, including the dynamic interfacial tension and the Marangoni stress, is derived using the concept of continuum surface force together with a coordinate transformation, and is then incorporated into the LBM through the body force model of Guo et al. [29]. An additional lattice Boltzmann equation is also introduced to describe the evolution of the axisymmetric temperature field, which is related to the interfacial tension by the equation of state. The proposed LBM is first validated against the analytical or benchmark solutions for the Rayleigh-Bénard convection in a vertical cylinder and the thermocapillary migration of an isolated droplet at negligibly small Reynolds and Marangoni numbers. It is found that the thermocapillary migration of an isolated droplet can reach the steady state for the Marangoni numbers up to $10^{3}$, and the terminal migration velocity diminishes with increasing the Marangoni number.

The axisymmetric LBM is then used to simulate the thermocapillary migration of two spherical droplets (with the leading droplet generally smaller than the trailing one, unless otherwise stated) in a constant applied temperature gradient along their line of centers, and the influence of the Marangoni number $(M a)$, initial distance between droplets $\left(S_{0}\right)$, and the radius ratio of the leading to trailing droplets $(\Lambda)$ on the migration process is systematically examined. It is observed that the droplet motion exhibits two different states with increasing $M a$. At low $M a$, the interaction between droplets is weak, and the trailing droplet can catch up with the leading one, eventually leading to a single droplet moving at a constant velocity. At moderate or high $M a$, the two droplets interact strongly and eventually reach the same velocity with a fixed distance $S$; the common velocity of the coupled droplets is slower than that of the bigger isolated droplet as a result of the thermal wakes behind the leading droplet, but faster than that of the smaller isolated droplet because of the isotherm-gathering effect just above the trailing droplet; and for the non-coalescing cases, the final distance between droplets increases with $M a$. The variation of the initial distance between droplets is found not to change the final state of the droplets albeit that it has a direct impact on the migration process. On the other hand, the radius ratio of the leading to trailing droplets can significantly affect the migration process and the final state: at high $M a$, the coupled droplets reach a common velocity only for small values of $\Lambda$, and a smaller $\Lambda$ corresponds to a shorter equilibrium time but a faster common velocity; at low $M a$, the final state of the droplets undergoes a transition from the coalescence to the non-coalescence with a fixed distance and finally to the non-coalescence with an increasing distance as $\Lambda$ increases. For $\Lambda=1$, no matter how small $M a$ is, the leading droplet migrates faster than the trailing one, and thus their distance keeps increasing during the migration. To experimentally reproduce the present results on the thermocapillary migration of two droplets, at the end we would like to offer several suggestions to experimentalists: (1) carefully design the experimental setup, e.g. the experimental fluids, droplet sizes, and the temperature gradients; (2) keep the symmetry of experimental system and droplet configuration; and (3) ensure that two droplets start to migrate simultaneously.

\section{Acknowledgements}

This work is financially supported by the Thousand Youth Talents Program for Distinguished Young Scholars, the National Natural Science Foundation of China (No. 51506168) and the China Postdoctoral Science Foundation (No. 2016M590943). 


\section{References}

[1] N. Young, J. Goldstein, M. Block, The motion of bubbles in a vertical temperature gradient, J. Fluid Mech. 6 (1959) 350-356.

[2] R. S. Subramanian, R. Balasubramaniam, The Motion of Bubbles and Drops in Reduced Gravity, Cambridge: Cambridge University Press, 2001.

[3] Z. Yin, Q. Li, Thermocapillary migration and interaction of drops: two non-merging drops in an aligned arrangement, J. Fluid Mech. 766 (2015) 436-467.

[4] J. L. Anderson, Droplet interactions in thermocapillary motion, Int. J. Multiphase Flow 11 (6) (1985) 813-824.

[5] Z. Yin, P. Gao, W. Hu, L. Chang, Thermocapillary migration of nondeformable drops, Phys. Fluids 20 (2008) 082101.

[6] H. Zhou, R. H. Davis, Axisymmetric thermocapillary migration of two deformable viscous drops, J. Coll. Interf. Sci. 181 (1) (1996) 60-72.

[7] C. Pozrikidis, Effect of inertia on the Marangoni instability of two-layer channel flow, Part I: numerical simulations, Journal of Engineering Mathematics 50 (2) (2004) 311-327.

[8] B. Samareh, J. Mostaghimi, C. Moreau, Thermocapillary migration of a deformable droplet, Int. J. Heat Mass Transf. 73 (2014) 616-626.

[9] H. Haj-Hariri, Q. Shi, A. Borhan, Thermocapillary motion of deformable drops at finite Reynolds and Marangoni numbers, Physics of Fluids 9 (4) (1997) 845-855.

[10] H. Liu, Q. Kang, C. R. Leonardi, B. D. Jones, S. Schmieschek, A. Narváez, J. R. Williams, A. J. Valocchi, J. Harting, Multiphase lattice Boltzmann simulations for porous media applications, Computational Geosciences 20 (4) (2016) 777-805.

[11] A. K. Gunstensen, D. H. Rothman, S. Zaleski, G. Zanetti, Lattice Boltzmann model of immiscible fluids, Phys. Rev. A 43 (8) (1991) 4320-4327.

[12] M. R. Swift, E. Orlandini, W. R. Osborn, J. M. Yeomans, Lattice Boltzmann simulations of liquid-gas and binary fluid systems, Phys. Rev. E 54 (5) (1996) 5041-5052.

[13] Y. Wang, C. Shu, H. Huang, C. Teo, Multiphase lattice Boltzmann flux solver for incompressible multiphase flows with large density ratio, Journal of Computational Physics 280 (2015) 404-423.

[14] Y. Wang, C. Shu, L. Yang, An improved multiphase lattice Boltzmann flux solver for three-dimensional flows with large density ratio and high Reynolds number, J. Comput. Phys. 302 (2015) 41-58.

[15] X. Shan, H. Chen, Lattice Boltzmann model for simulating flows with multiple phases and components, Phys. Rev. E 47 (3) (1993) 1815-1819. 
[16] X. He, S. Chen, R. Zhang, A lattice Boltzmann scheme for incompressible multiphase flow and its application in simulation of Rayleigh-Taylor instability, J. Comput. Phys. 152 (2) (1999) 642-663.

[17] J. Zhang, Lattice Boltzmann method for microfluidics: models and applications, Microfluid. Nanofluid. $10(1)(2011) 1-28$.

[18] H. Liu, Y. Zhang, A. J. Valocchi, Modeling and simulation of thermocapillary flows using lattice Boltzmann method, J. Comput. Phys. 231 (12) (2012) 4433-4453.

[19] H. Liu, Y. Zhang, Modeling thermocapillary migration of a microfluidic droplet on a solid surface, J. Comput. Phys. 280 (2015) 37-53.

[20] H. Liu, A. J. Valocchi, Y. Zhang, Q. Kang, A phase-field-based lattice-Boltzmann finite-difference model for simulating thermocapillary flows, Phys. Rev. E 87 (2013) 013010.

[21] H. Liu, A. J. Valocchia, Y. Zhang, Q. Kang, Lattice Boltzmann phase-field modeling of thermocapillary flows in a confined microchannel, J. Comput. Phys. 256 (2014) 334-356.

[22] S. V. Lishchuk, C. M. Care, I. Halliday, Lattice Boltzmann algorithm for surface tension with greatly reduced microcurrents, Phys. Rev. E 67 (2003) 036701.

[23] I. Halliday, A. P. Hollis, C. M. Care, Lattice Boltzmann algorithm for continuum multicomponent flow, Phys. Rev. E 76 (2007) 026708.

[24] J. U. Brackbill, D. B. Kothe, C. Zemach, A continuum method for modeling surface tension, J. Comput. Phys. 100 (2) (1992) 335-354.

[25] J. G. Zhou, Axisymmetric lattice Boltzmann method, Phys. Rev. E 78 (2008) 036701.

[26] S. Srivastava, P. Perlekar, J. H. M. ten Thije Boonkkamp, N. Verma, F. Toschi, Axisymmetric multiphase lattice Boltzmann method, Phys. Rev. E 88 (2013) 013309.

[27] P. Lallemand, L.-S. Luo, Theory of the lattice Boltzmann method: Dispersion, dissipation, isotropy, Galilean invariance, and stability, Phys. Rev. E 61 (6) (2000) 6546-6562.

[28] H. Liu, L. Wu, Y. Ba, G. Xi, Y. Zhang, A lattice Boltzmann method for axisymmetric multicomponent flows with high viscosity ratio, J. Comput. Phys.(submitted).

[29] Z. Guo, C. Zheng, B. Shi, Discrete lattice effects on the forcing term in the lattice Boltzmann method, Phys. Rev. E 65 (2002) 046308.

[30] Z. Chai, T. S. Zhao, Effect of the forcing term in the multiple-relaxation-time lattice Boltzmann equation on the shear stress or the strain rate tensor, Phys. Rev. E 86 (2012) 016705.

[31] H. Liu, A. J. Valocchi, C. Werth, Q. Kang, M. Oostrom, Pore-scale simulation of liquid CO2 displacement of water using a two-phase lattice Boltzmann model, Adv. Water Resour. 73 (2014) 144-158. 
[32] M. Latva-Kokko, D. H. Rothman, Diffusion properties of gradient-based lattice Boltzmann models of immiscible fluids, Phys. Rev. E 71 (2005) 056702.

[33] H. Liu, Y. Zhang, Droplet formation in microfluidic cross-junctions, Phys. Fluids 23 (8) (2011) 082101.

[34] Q. Li, Y. L. He, G. H. Tang, W. Q. Tao, Lattice Boltzmann model for axisymmetric thermal flows, Phys. Rev. E 80 (2009) 037702.

[35] A. J. C. Ladd, Numerical simulations of particulate suspensions via a discretized Boltzmann equation. (Part I \& II), J. Fluid Mech. 271 (1994) 285-339.

[36] T. Zhang, B. Shi, Z. Guo, Z. Chai, J. Lu, General bounce-back scheme for concentration boundary condition in the lattice-Boltzmann method, Phys. Rev. E 85 (2012) 016701.

[37] S. Lang, A. Vidal, A. Acrivos, Buoyancy-driven convection in cylindrical geometries, J. Fluid Mech. 36 (1969) 239-256.

[38] A. Lemembre, J.-P. Petit, Laminar natural convection in a laterally heated and upper cooled vertical cylindrical enclosure, Int. J. Heat Mass Transf. 41 (16) (1998) 2437-2454.

[39] L. Zheng, B. Shi, Z. Guo, C. Zheng, Lattice Boltzmann equation for axisymmetric thermal flows, Comput. Fluids 39 (6) (2010) 945-952.

[40] Y. Wang, C. Shu, C. J. Teo, L. M. Yang, A fractional-step lattice Boltzmann flux solver for axisymmetric thermal flows, Numerical Heat Transfer, Part B: Fundamentals 69 (2) (2016) 111-129.

[41] N. Shankar, R. S. Subramanian, The stokes motion of a gas bubble due to interfacial tension gradients at low to moderate marangoni numbers, J. Colloid Interface Sci. 123 (2) (1988) 512-522.

[42] Y. Wang, X. Lu, L. Zhuang, Z. Tang, W. Hu, Numerical simulation of drop marangoni migration under microgravity, Acta Astronaut. 54 (5) (2004) 325-335.

[43] M. Hill, On a spherical vortex, Proc. R. Soc. Lond. 55 (1894) 219-224. 
Table 1: Comparison of the maximum velocity in the flowfield.

\begin{tabular}{cc|cc}
\hline \hline & & Upflow & Downflow \\
\hline \multirow{4}{*}{$R a=5000$} & Ref. [38] & 0.353 & 0.353 \\
& Ref. [40] & 0.354 & 0.351 \\
& Present LBM & 0.3526 & 0.3529 \\
\hline
\end{tabular}




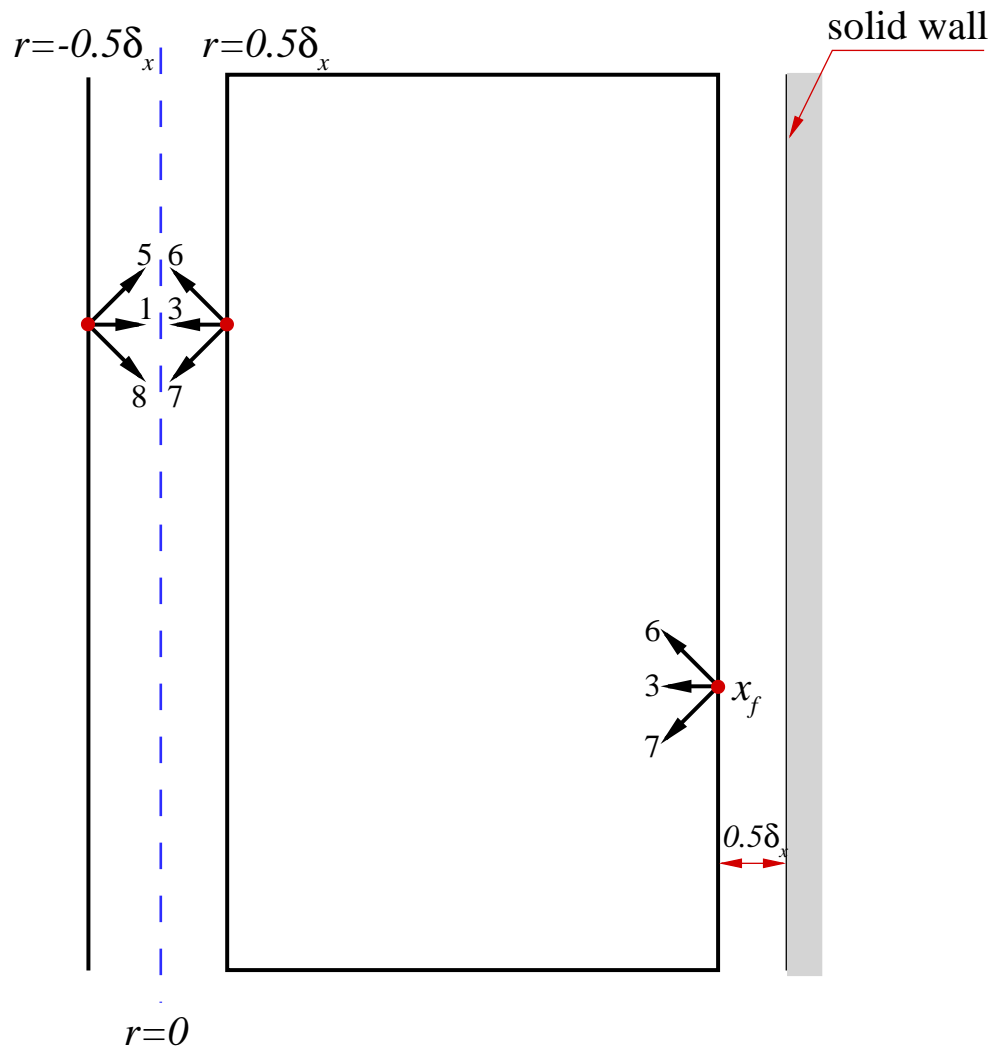

Figure 1: (Color Online) Schematic diagram of the computational geometry and boundary conditions. $r=0$ represents the symmetric axis. 


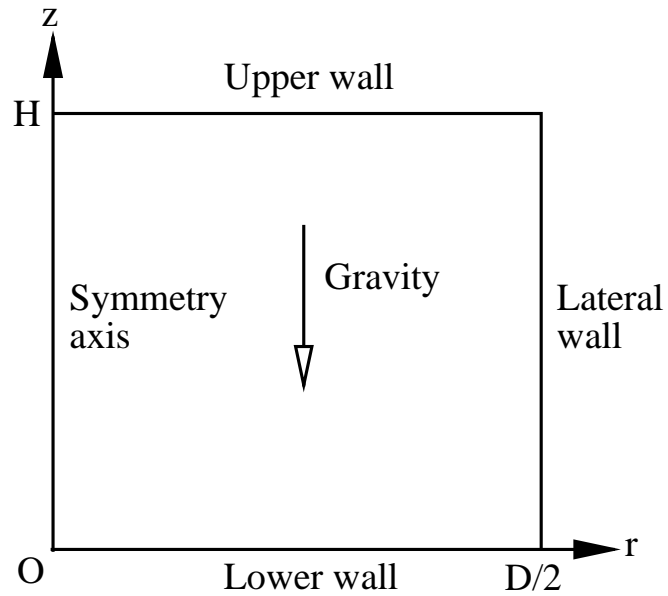

Figure 2: Sketch of Rayleigh-Bénard convection in a vertical cylinder. 

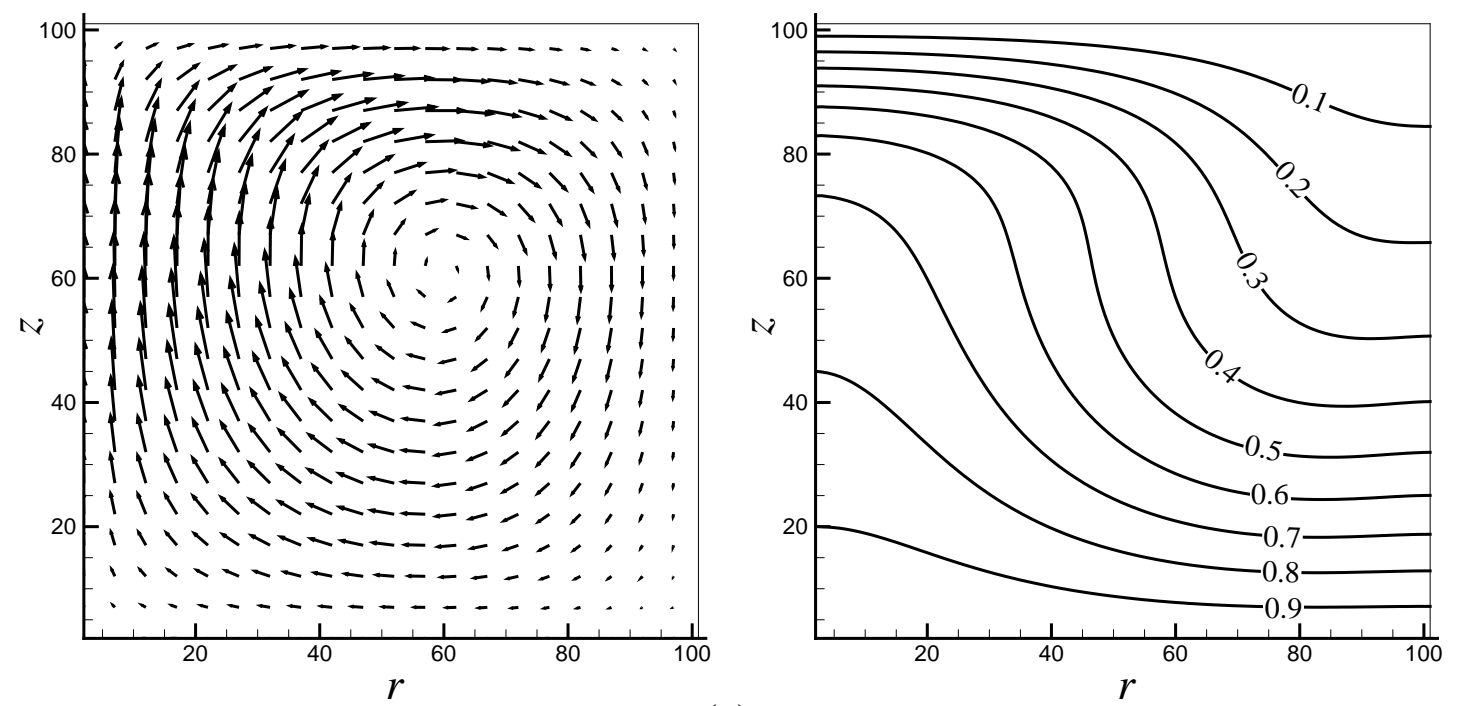

(a)
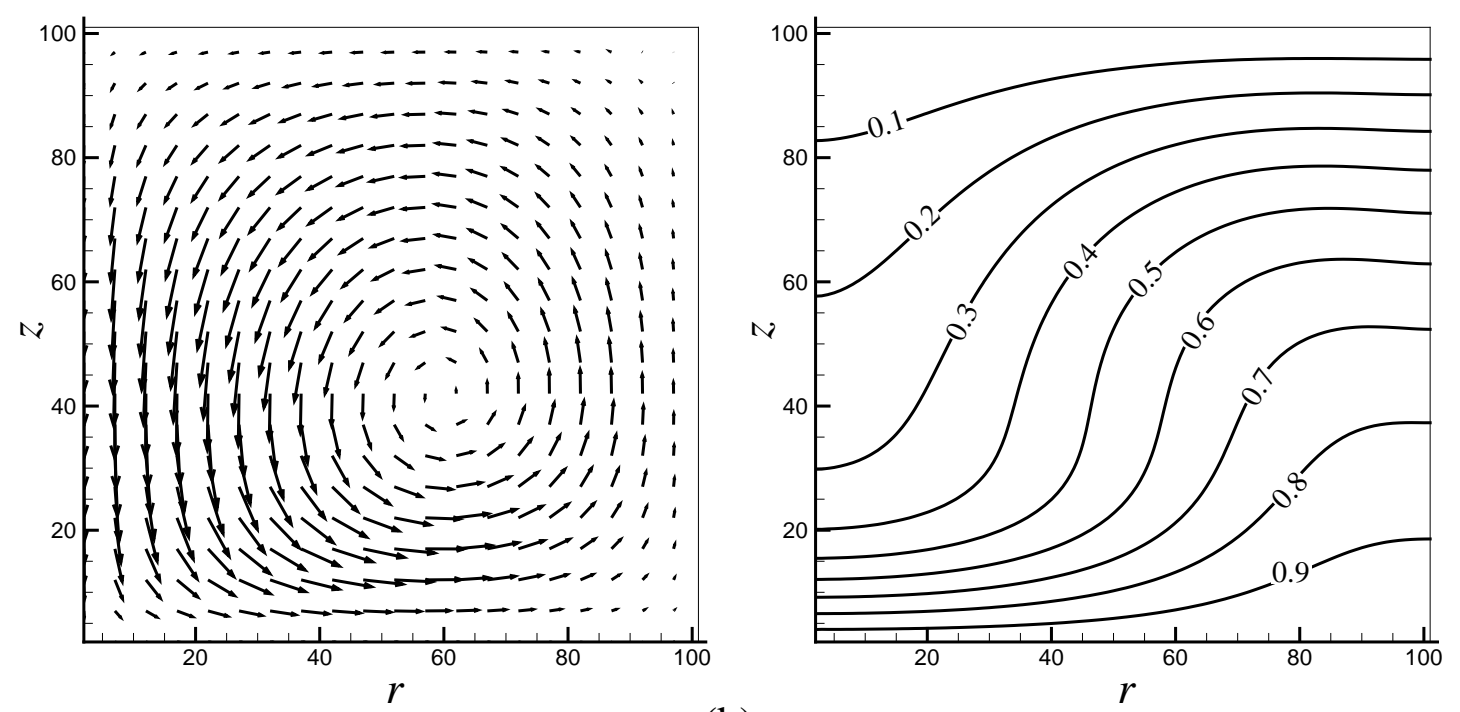

(b)

Figure 3: Velocity vectors (left) and isotherms (right) at $R a=5000$ for (a) an upflow and (b) a downflow in the center of the cylinder. Note that the velocity vectors are shown at every fifth grid point. 


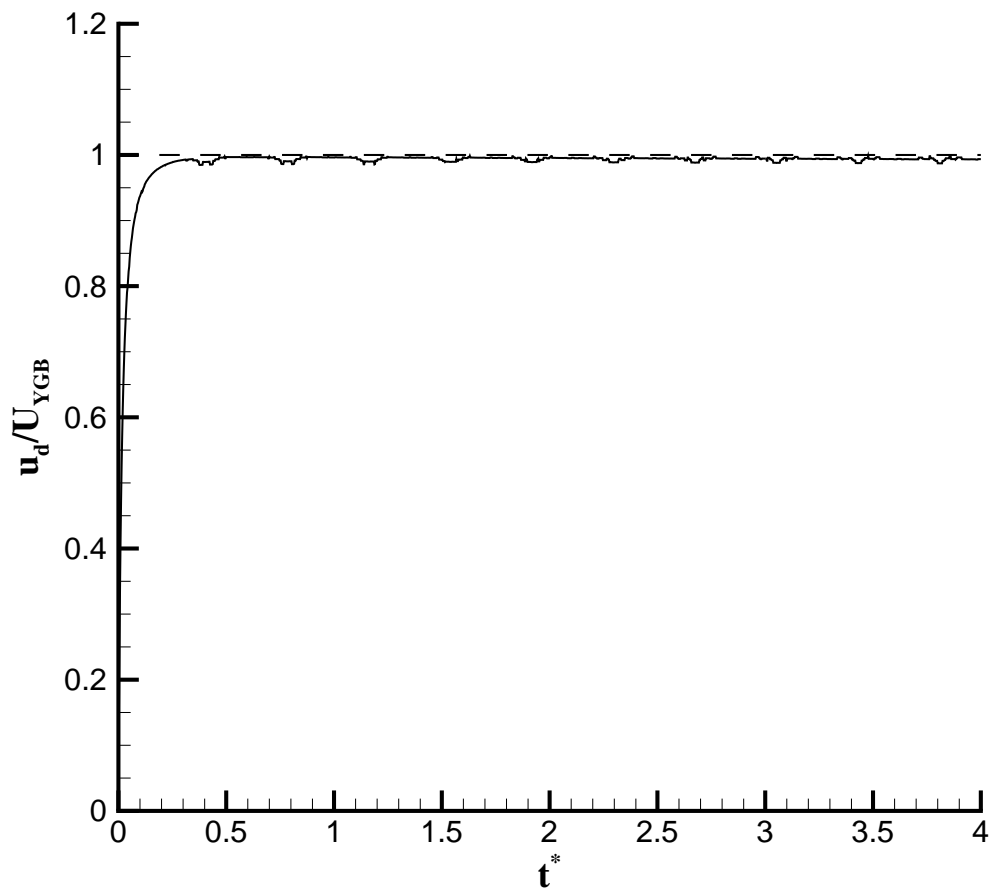

Figure 4: Time evolution of normalized migration velocity of a spherical droplet at $R e=M a=0.1$. The dashed line represents the analytical prediction in the limit of vanishing Reynolds and Marangoni numbers, while the solid line is the present simulation result. 


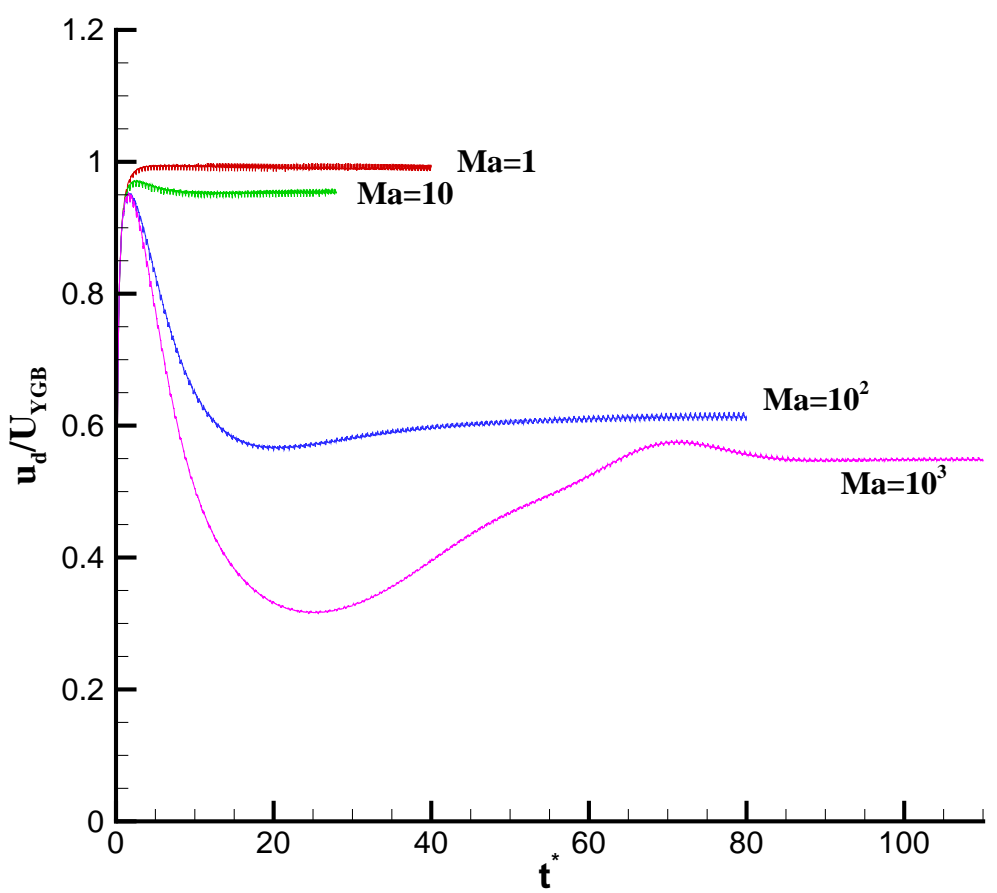

Figure 5: (Color Online) Time evolution of the droplet migration velocity for different values of $M a$ at $R e=1$. The droplet migration velocity $u_{d}$ is normalized by the YGB velocity. 

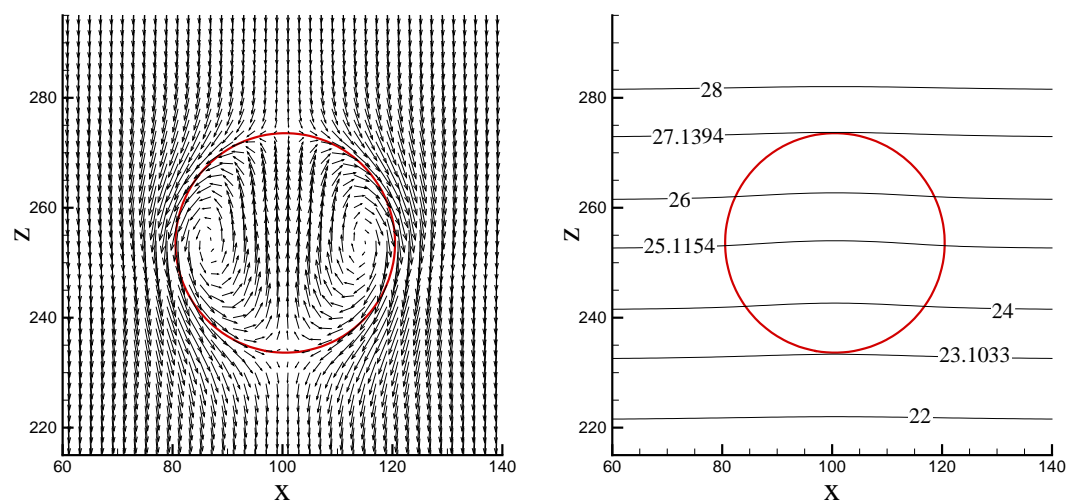

(a) $\mathrm{Ma}=1$
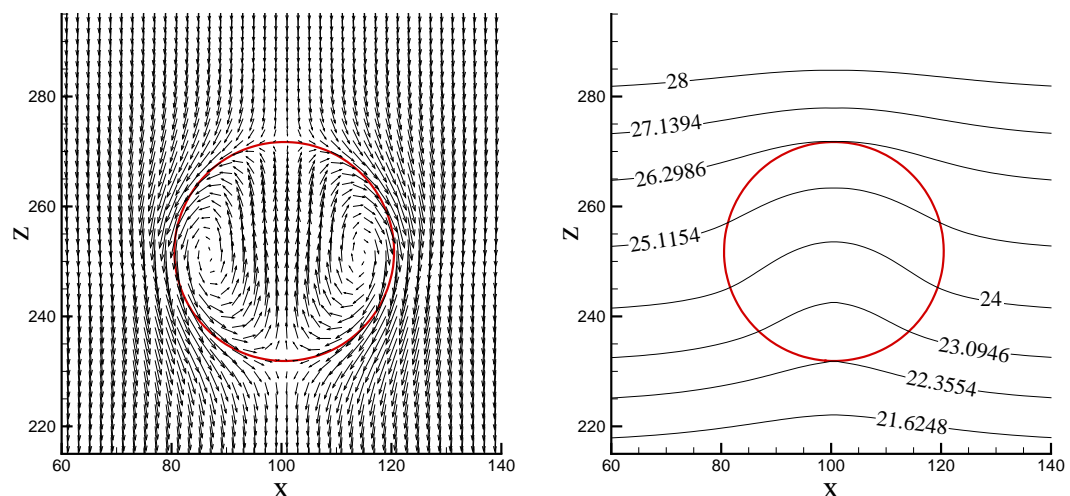

(b) $\mathrm{Ma}=10$
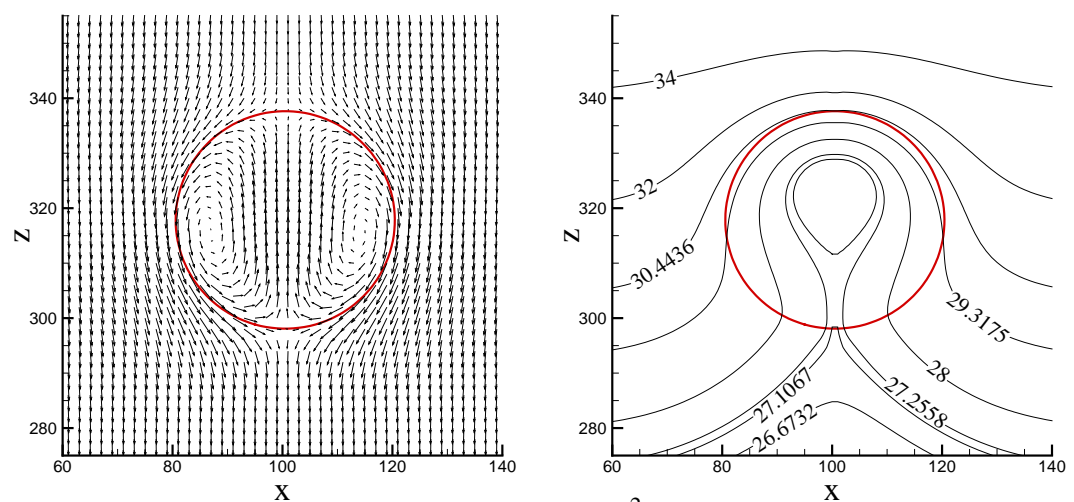

(c) $\mathrm{Ma}=10^{2}$
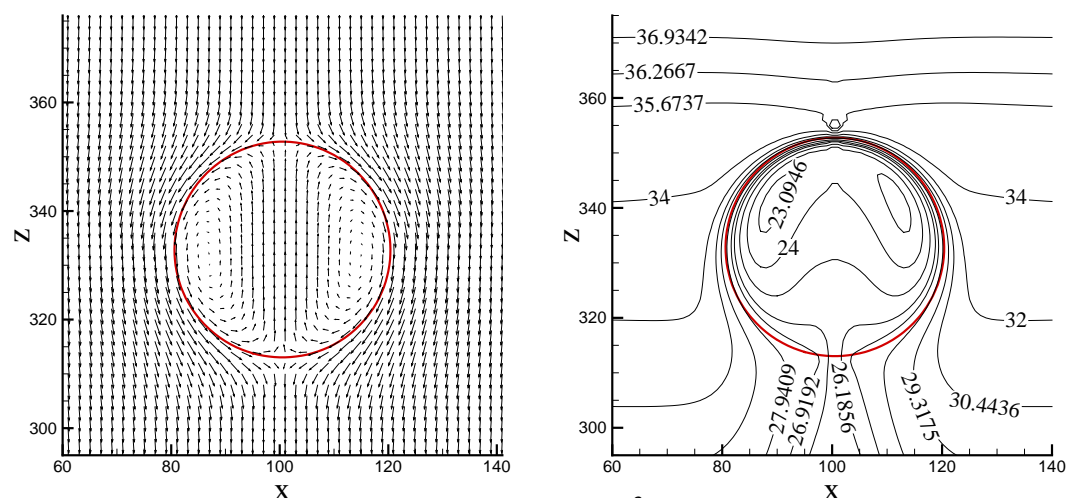

(d) $\mathrm{Ma}=10^{3}$

Figure 6: (Color Online) Velocity vectors (left) and isotherms (right) around the droplet in a reference frame moving with the droplet for (a) $M a=1$, (b) $M a=10$, (c) $M a=10^{2}$, and (d) $M a=10^{3}$. The red solid lines represent the droplet profiles. The abscissa $x$ is defined by $x=r+100$. 


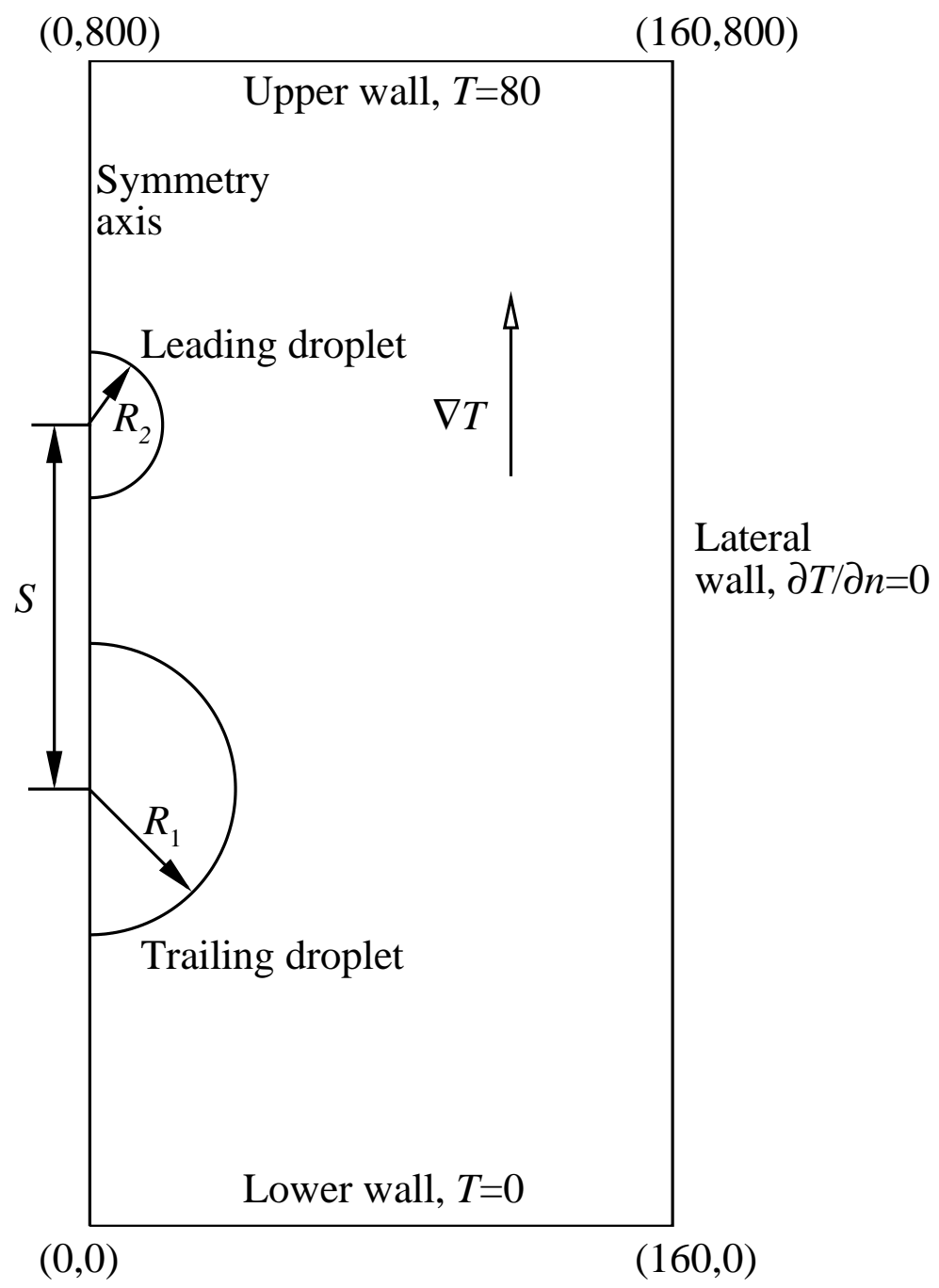

Figure 7: Schematics of the computational geometry and boundary conditions for the thermocapillary migration of two deformable droplets. 


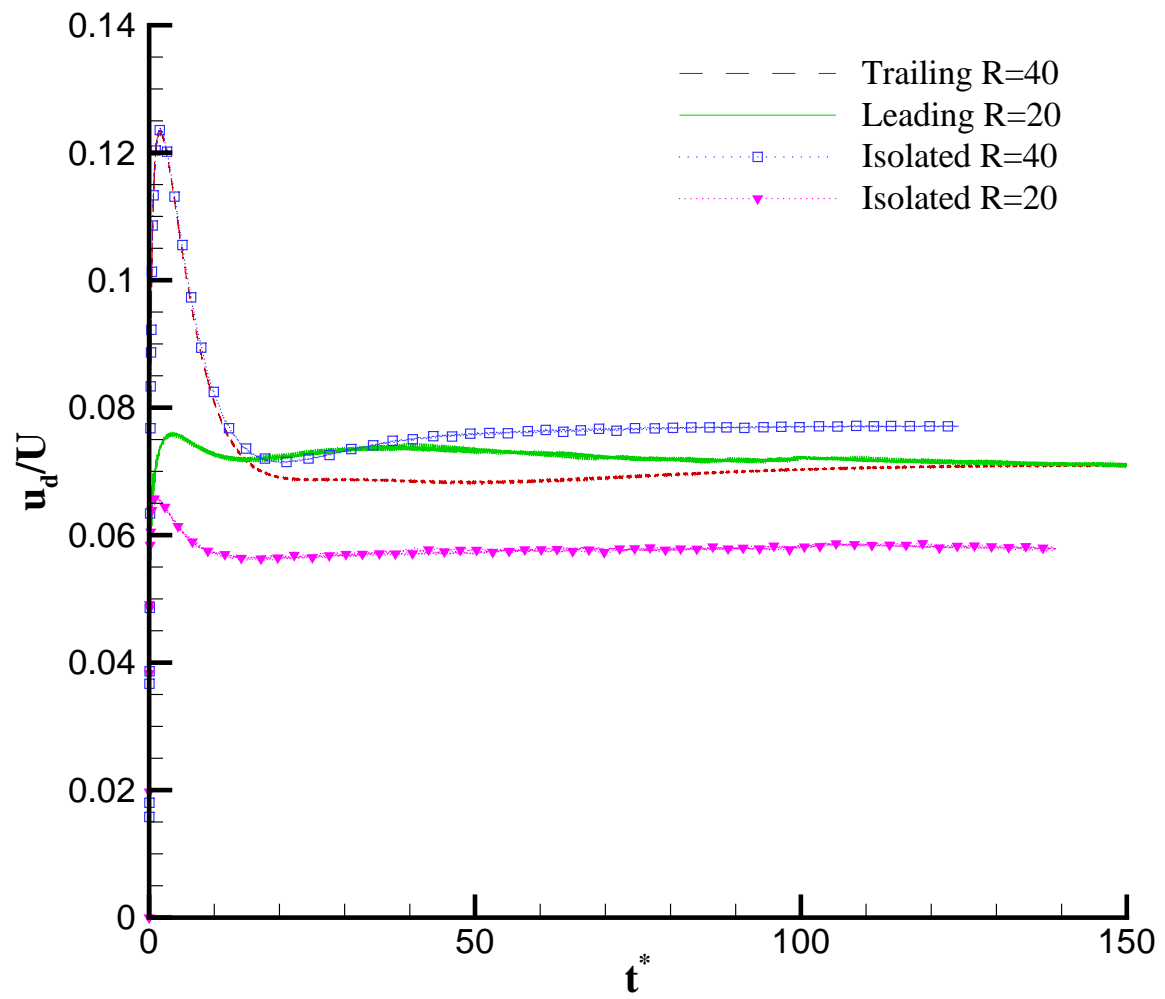

Figure 8: (Color Online) Time evolution of the droplet migration velocities for $M a=100, R_{2}=0.5 R_{1}$ and $S_{0}=2.5 R_{1}$. The droplet migration velocity $u_{d}$ is normalized by the characteristic velocity $U$, and the dimensionless time is defined as $t^{*}=U t / R_{1}$. 
(a)

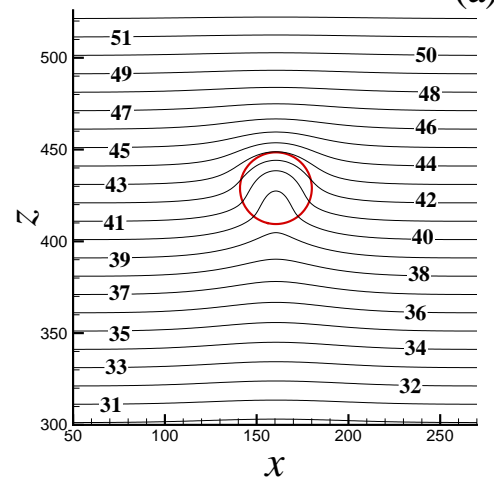

(b)

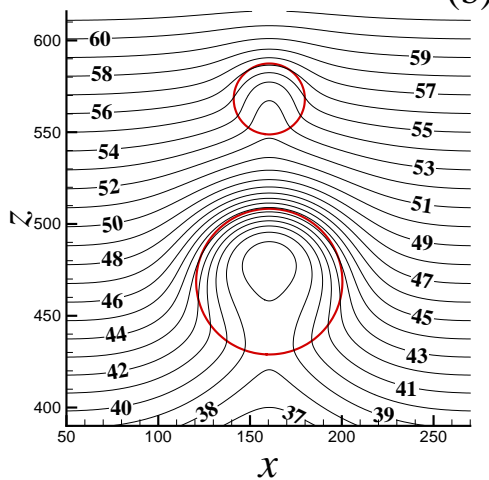

(c)

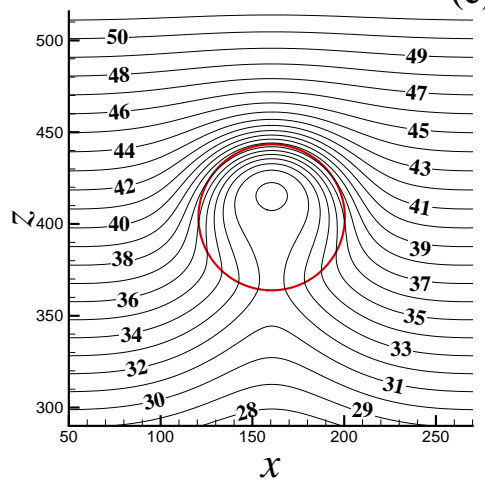

Figure 9: (Color Online) Comparison of temperature fields between the binary droplets and isolated droplets in the final state for $M a=100$ : (a) isolated droplet with $R=20$, (b) binary droplets with $R_{1}=40, R_{2}=20$ and $S_{0}=2.5 R_{1},(\mathrm{c})$ isolated droplet with $R=40$. The abscissa $x$ is defined by $x=r+160$. 


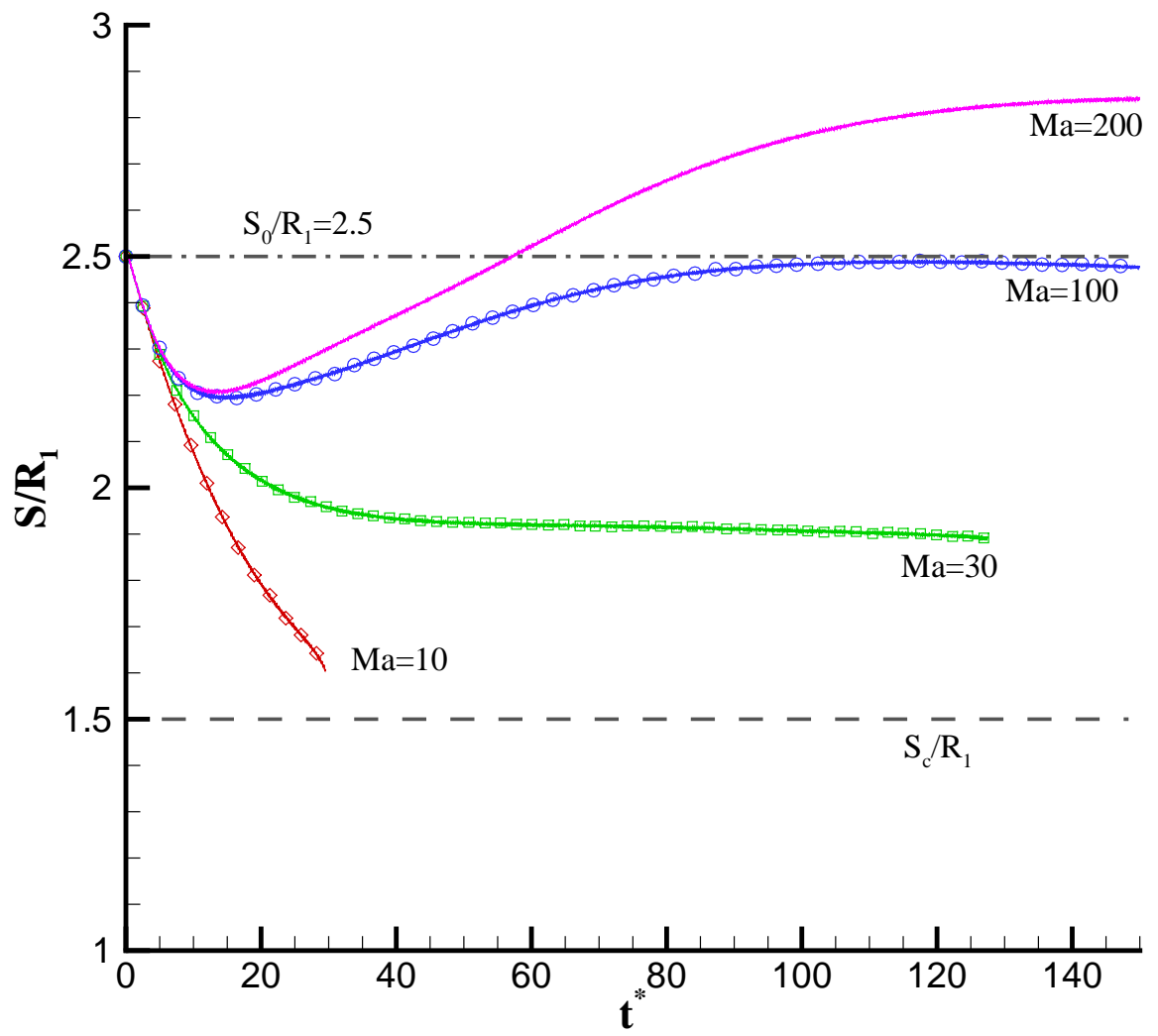

Figure 10: (Color Online) Time evolution of the droplet distance $S$ for different values of $M a$ at $R_{2}=0.5 R_{1}$ and $S_{0}=2.5 R_{1}$. The droplet distance $S$ is normalized by $R_{1}$, and the dimensionless time is defined as $t^{*}=U t / R_{1}$. Note that $S_{c}$ is the critical droplet distance, below which two undeformed spherical droplets will merge together. 

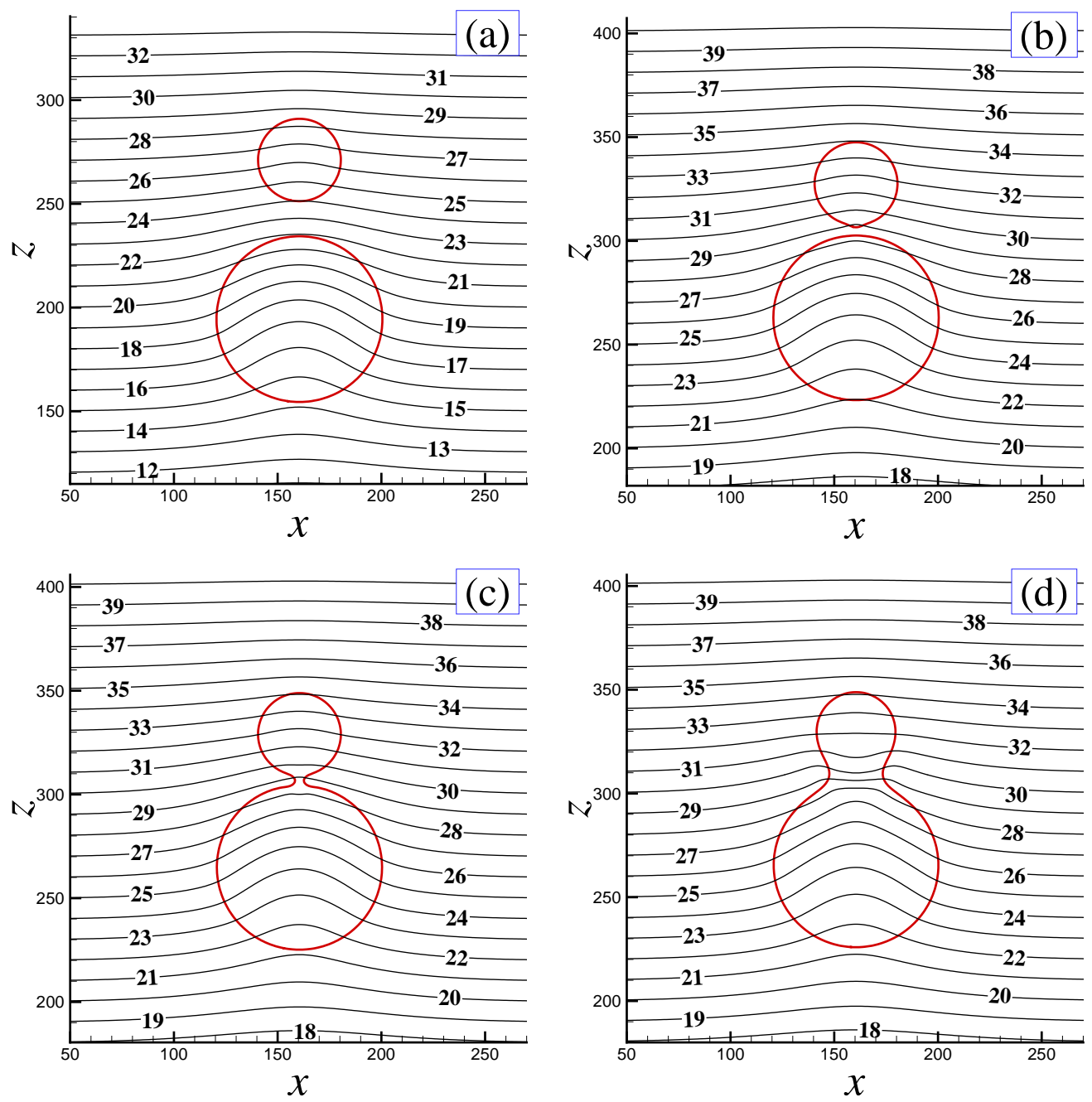

(e)

(f)
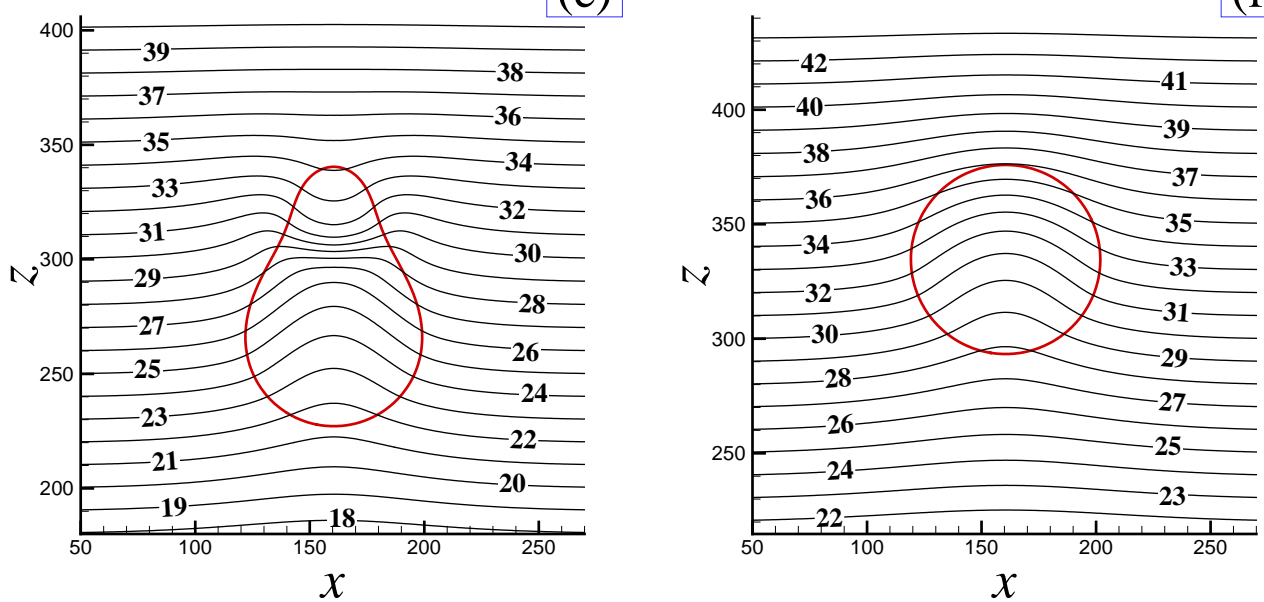

Figure 11: (Color Online) Snapshots of the droplet shapes (represented by red solid lines) and the isotherms around the droplets for $M a=10, R_{2}=0.5 R_{1}$ and $S_{0}=2.5 R_{1}$. The abscissa $x$ is defined by $x=r+160$. 


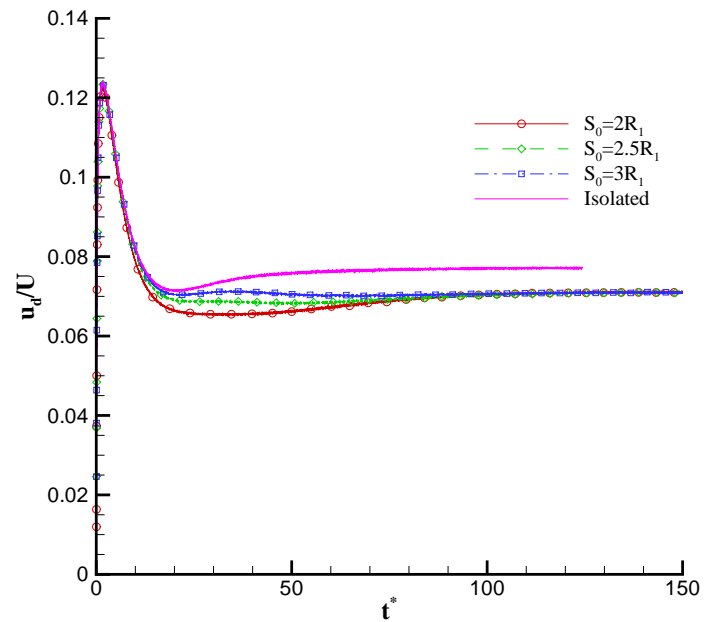

(a)

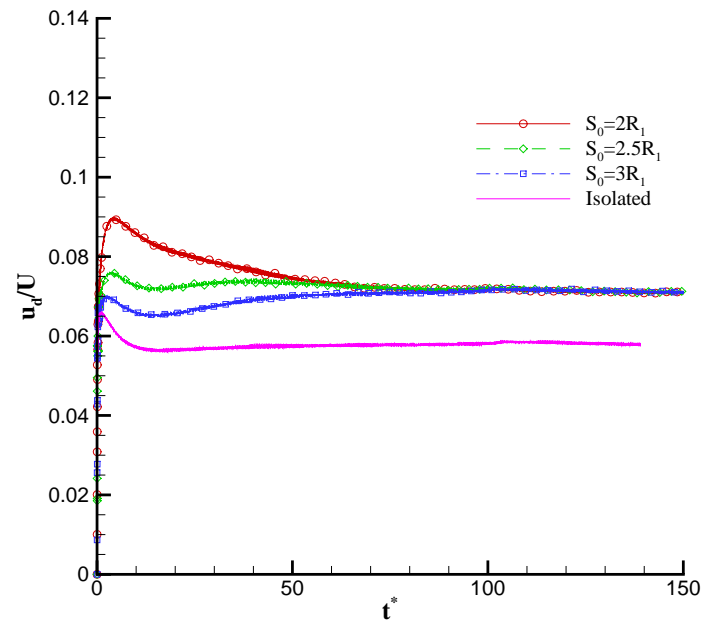

(b)

Figure 12: (Color Online) Time evolution of the droplet migration velocities at different $S_{0}$ for $M a=100$ and $R_{2}=0.5 R_{1}:(a)$ trailing droplet, (b) leading droplet. The droplet migration velocity $u_{d}$ is normalized by the characteristic velocity $U$, and the dimensionless time is defined as $t^{*}=U t / R_{1}$. 


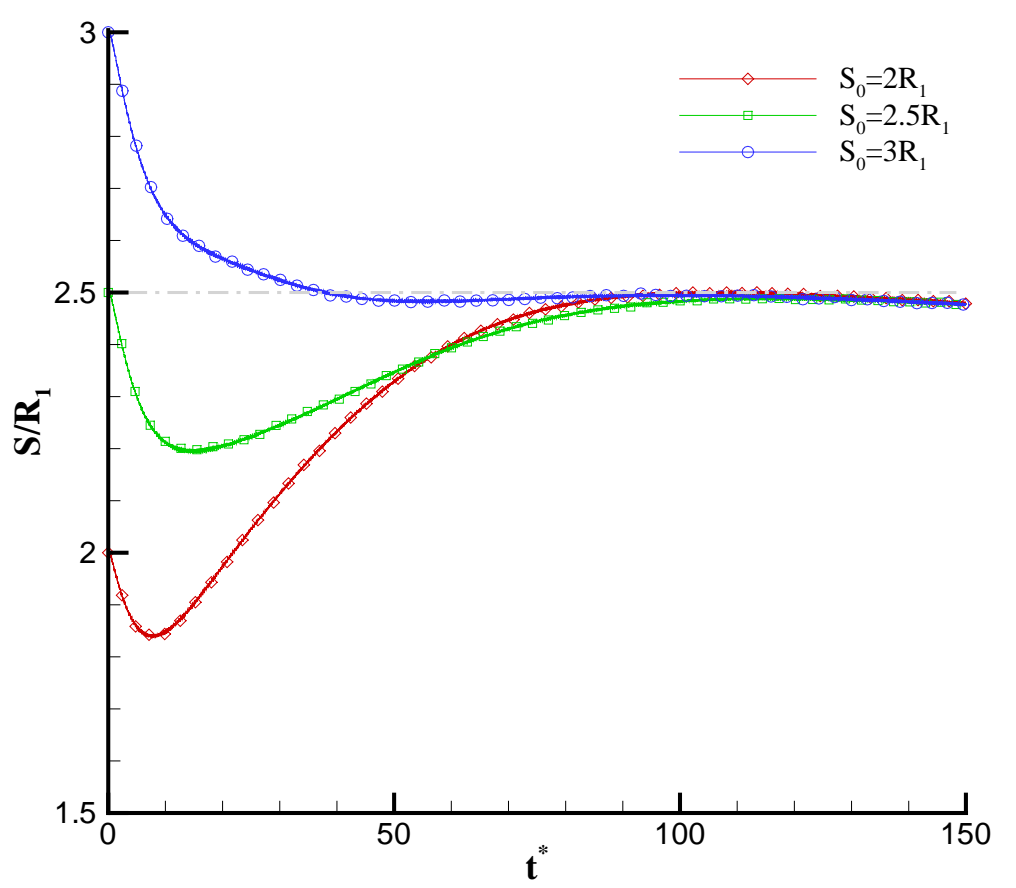

Figure 13: (Color Online) Time evolution of the droplet migration velocity at different $S_{0}$ for $M a=100$ and $R_{2}=0.5 R_{1}$. The droplet distance $S$ is normalized by $R_{1}$, and the dimensionless time is defined as $t^{*}=U t / R_{1}$. 


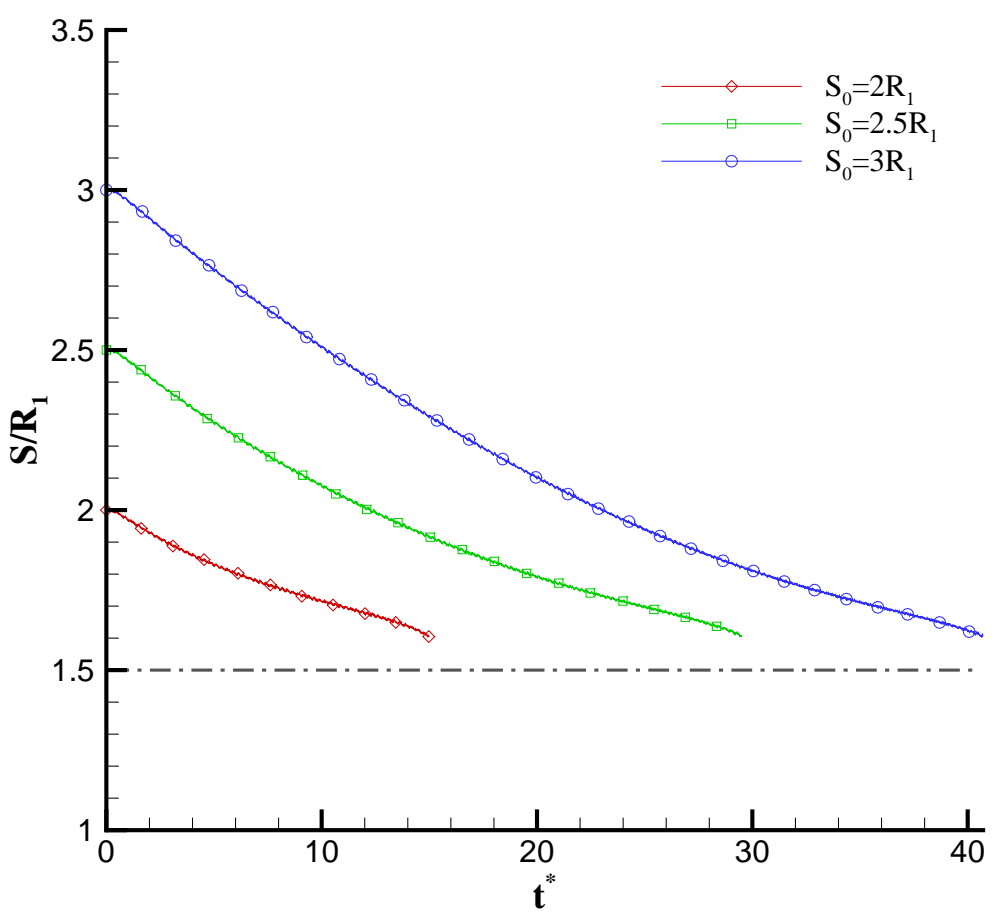

Figure 14: (Color Online) Time evolution of the droplet migration velocity at different $S_{0}$ for $M a=10$ and $R_{2}=0.5 R_{1}$. The droplet distance $S$ is normalized by $R_{1}$, and the dimensionless time is defined as $t^{*}=U t / R_{1}$. Note that the dash-dotted line represents the critical droplet distance, below which two undeformed spherical droplets will merge together. 


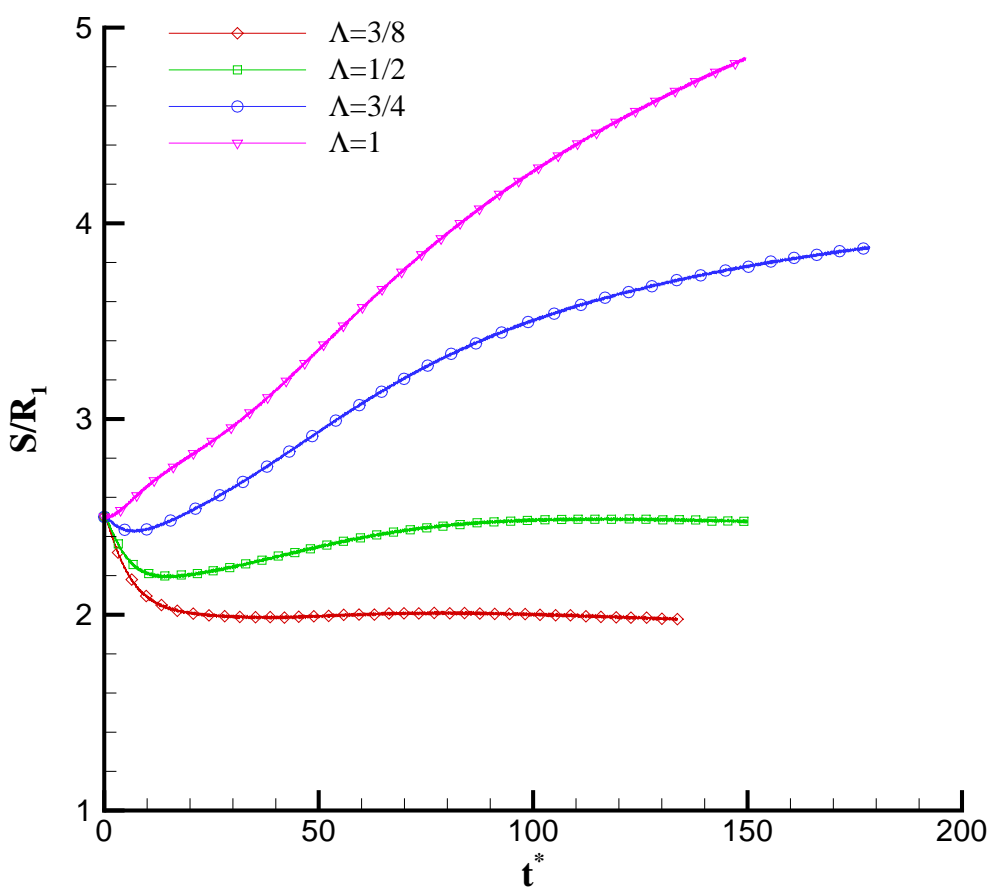

Figure 15: (Color Online) Time evolution of the droplet distance $S$ for different $\Lambda$ at $M a=100$ and $S_{0}=2.5 R_{1}$. The droplet distance $S$ is normalized by $R_{1}$, and the dimensionless time is defined as $t^{*}=U t / R_{1}$. 


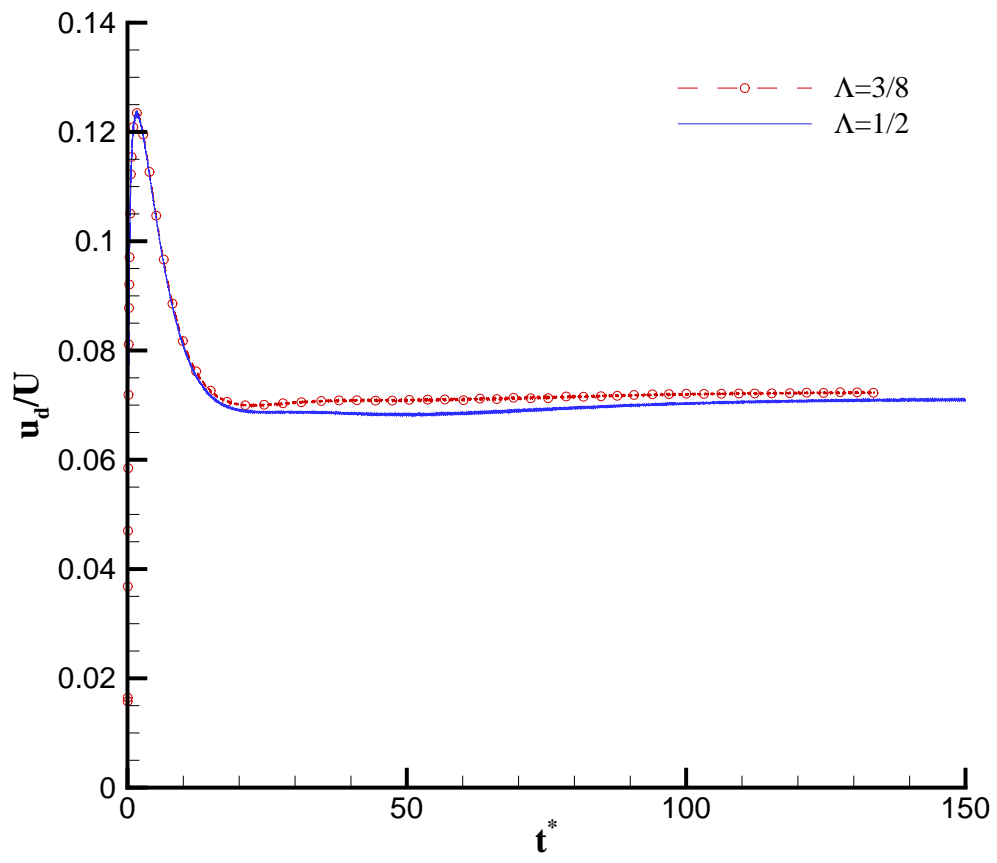

Figure 16: (Color Online) Time evolution of the migration velocity of the trailing droplet for $\Lambda=3 / 8$ and $1 / 2$ at $M a=100$ and $S_{0}=2.5 R_{1}$. The droplet migration velocity $u_{d}$ is normalized by the characteristic velocity $U$, and the dimensionless time is defined as $t^{*}=U t / R_{1}$. 


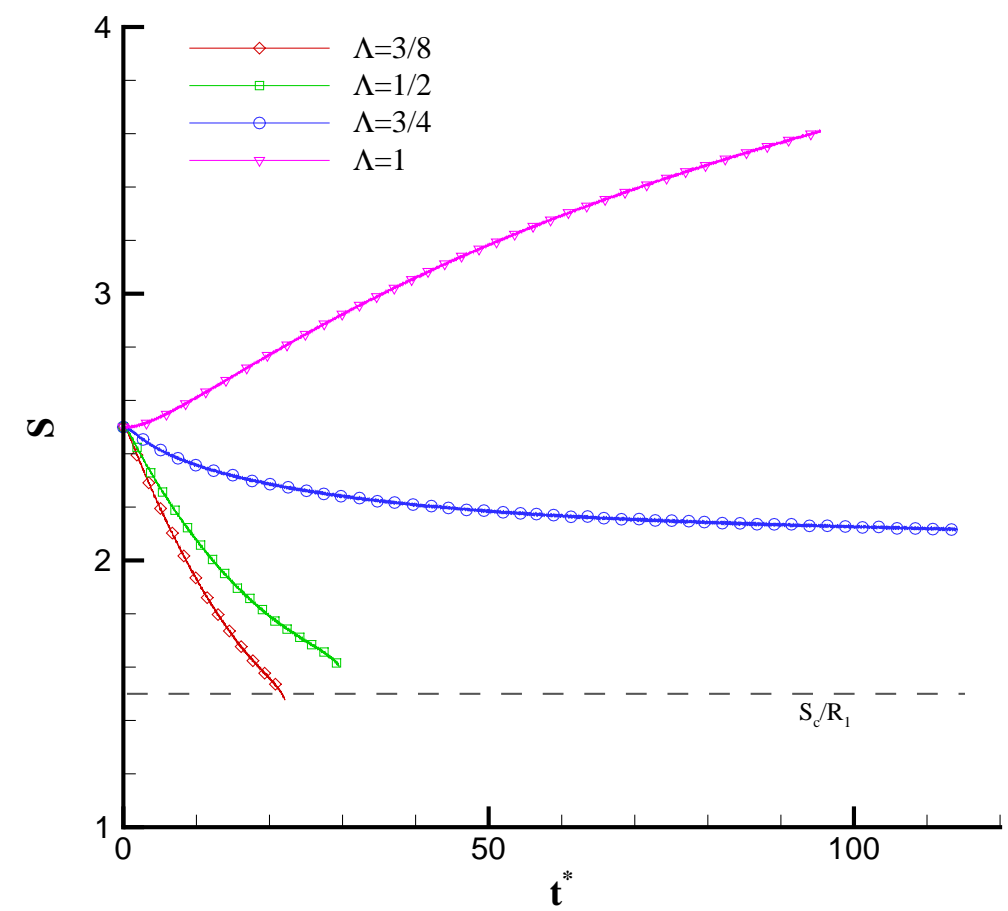

Figure 17: (Color Online) Time evolution of the droplet distance $S$ for different $\Lambda$ at $M a=10$ and $S_{0}=2.5 R_{1}$. The droplet distance $S$ is normalized by $R_{1}$, and the dimensionless time is defined as $t^{*}=U t / R_{1}$. Note that $S_{c}$ is the critical droplet distance, below which two undeformed spherical droplets will merge together. 